\title{
Three polymorphisms of renin-angiotensin system and preeclampsia risk
}

\author{
Chen Wang $^{1} \cdot$ Xiao Zhou $^{1} \cdot$ Huai Liu ${ }^{1} \cdot$ Shuhui Huang ${ }^{1}$ \\ Received: 18 June 2020 / Accepted: 6 October 2020 / Published online: 23 November 2020 \\ (C) The Author(s) 2020
}

\begin{abstract}
Purpose Some data suggest an association between the single nucleotide polymorphisms AGT T704C, ACE I/D, and AT1R A1166C and preeclampsia, but overall, the data are conflicting; the aim of our study was to discover a more stable and reliable association between these polymorphisms and PE risk.

Methods A comprehensive literature search for this meta-analysis was conducted. Odds ratios (OR) and 95\% confidence intervals (CIs) were calculated to evaluate the strength, and heterogeneity test was conducted. Trial sequential analysis was also performed.

Results A total of forty studies were finally included in our meta-analysis. The AGT T704C polymorphism was associated with $\mathrm{PE}$ risk in three genetic models (dominant $\mathrm{OR}=1.33,95 \% \mathrm{CI}=1.12-1.59$; heterozygote $\mathrm{OR}=1.26,95 \% \mathrm{CI}=1.05-1.52$; homozygote $\mathrm{OR}=1.44,95 \% \mathrm{CI}=1.14-1.83$ ). No heterogeneity was observed in the three genetic models for the ACE I/D polymorphism. For subgroup analysis by geography, no significant association was detected. Significant associations were observed in mixed race, early-onset, late-onset, and more than 200 subgroups for the AT1R A1166C polymorphism; however, only one study was analyzed in these subgroups.

Conclusions Our results indicated the AGT T704C and ACE I/D polymorphisms were associated with an increased risk of PE. Increased risks were also observed for the two polymorphisms in subgroups including Asians, Europeans, Caucasoid, and Mongoloid. Moreover, an increased PE risk with the ACE I/D polymorphism in the severe PE population was also detected. Regarding the AT1R A1166C polymorphism, weak associations were observed, but further studies are required.
\end{abstract}

Keywords Polymorphism · AGT T704C · ACE I/D · AT1R A1166C · preeclampsia · risk

\section{Introduction}

Preeclampsia (PE) is a common complication of pregnancy characterized by hypertension and proteinuria after 20 weeks of gestation [1]; it is one of major causes of maternal-fetal and

Chen Wang and Xiao Zhou contributed equally to this work.

Electronic supplementary material The online version of this article (https://doi.org/10.1007/s10815-020-01971-8) contains supplementary material, which is available to authorized users.

Shuhui Huang

shhuang86@163.com

1 Department of Gynecology, Maternal and Child Health Affiliated Hospital of Nanchang University, Nanchang City, Jiangxi, People's Republic of China neonatal morbidity and mortality worldwide [2]. Knowing the risk factors for preeclampsia is critical for its prevention and treatment. Genetic factors play an important role in the genesis and development of PE and the genetic susceptibility to preeclampsia has generated great attention; the T allele of AGT may play a role in the pathogenesis of PE reported by Aung et al. [3],which indicated the gene polymorphisms in the renin-angiotensin-aldosterone system (RAAS) may be risk factors to PE.

During normal pregnancy, the upregulation of renin and aldosterone triggered by the stimulation of the RAAS system maintains the balance of blood volume and blood pressure [4]; however, for PE subjects, depression of the RAAS system with increased vascular resistance was observed, suggesting its crucial role in the pathogenesis of PE [5]. Angiotensin (AGT), angiotensin converting enzyme (ACE), and angiotensin II type 1 receptor (AT1R) are the three pivotal nodes in the RAAS system. The cleavage of AGT by renin contributes to 
the generation of angiotensin I, then ACE catalyzes the conversion of angiotensin I to a physiologically active angiotensin II. Finally, by binding to AT1R, angiotensin II regulates blood pressure by controlling sodium excretion [6]. Therefore, studies regarding the associations between single nucleotide polymorphisms in RAAS genes and PE risk are essential.

Associations between the polymorphisms of AGT T704C (the substitution of $\mathrm{C}$ to $\mathrm{T}$ at exon 2), ACE I/D (the insertion or deletion of an Alu 289 base pair sequence at intron 16), and AT1R A1166C (the change from C to A at 3'UTR) have been widely studied with conflicting results. To our best knowledge, differences in the geographic regions, ethnicity, and sample size could be reasons for the inconsistency. Moreover, the number of gestational weeks and the severity of PE have been reported to be associated with RAAS susceptibility gene polymorphisms [7-9], but these were not discussed in previous meta-analyses. Therefore, we conducted a comprehensive meta-analysis with trial sequential analysis to investigate the associations between the polymorphisms AGT T704C, ACE I/D, AT1R A1166C, and PE risk.

\section{Methods}

\section{Literature search}

PubMed, Embase, Google scholar, China National Knowledge Internet (CNKI), Baidu Scholar, Wan Fang, and VIP databases were comprehensively searched for studies regarding the associations between ACE insertion/deletion, AGT T704, and AT1R A1166C polymorphisms and preeclampsia susceptibility up to May 13, 2018. No language limitation was set. The following key words were used to discover relevant articles: "angiotensin-converting enzyme," "angiotensin," "angiotensin II type 1 receptor," "ACE," "AGT," "AT1R," "polymorphism," "variant," "single nucleotide polymorphism," "SNP," "preeclampsia," "PE," "hypertension," and "pregnancy-induced hypertension syndrome." The references of relevant studies were also screened by hand to identify potential studies. Our work was based on the Preferred Reporting Items for Systematic Reviews and Meta-analyses (PRISMA) statement [10] (Fig. 1).

\section{Inclusion and exclusion criteria}

The inclusion criteria for studies were as follows: (1) casecontrol studies discussing the relationship between ACE I/D, AGT T704C, AT1R A1166C polymorphisms, and preeclampsia risk; (2) the diagnostic criteria for preeclampsia were defined as gestational hypertension, assessed as SBP > $140 \mathrm{mmHg}$, DBP > $90 \mathrm{mmHg}$, and/or rise in SBP > $30 \mathrm{mmHg}$ or DBP $>15 \mathrm{mmHg}$ on at least two occasions $6 \mathrm{~h}$ apart, following 20 weeks of gestation, with marked proteinuria (>
$300 \mathrm{mg} / 24 \mathrm{~h}$ ), or $>2+$ proteinuria as tested by the dipstick method $[5,11,12]$; (3) the frequencies of the related polymorphisms in patients and controls could be retrieved to calculate odds ratio with $95 \%$ confidence intervals and to assess HardyWeinberg equilibrium. The exclusion criteria were (1) reviews or case reports or animal studies; (2) studies without reporting detailed genotype data; and (3) duplicated studies.

\section{Data extraction and quality assessment}

The following information from eligible studies were extracted by the first two authors: the first author' name, publication year, country, geography, ethnicity, PE maternal age, gestational weeks, PE degree, the genotype distributions and alleles in the patient and control groups, the result of the HardyWeinberg equilibrium, and the scores for quality assessment. For gestational weeks, early-onset PE was defined as gestational age (GA) between 20 and 33 weeks and 6 days, and late-onset PE was defined as GA 34 weeks and above. Severe PE was defined as severe hypertension (blood pressure $\geq 160$ / $110 \mathrm{mmHg}$ at least twice in a 24-h period) and/or severe proteinuria $(5 \mathrm{~g} / 24 \mathrm{~h})$, or as hypertension with multiorgan involvement including fetal growth restriction or HELLP syndrome (hemolysis, elevated liver enzymes, and low platelet count) [13]. Any disagreement was resolved by group discussion with the corresponding author. The qualities of included studies were assessed by all the authors in accordance with the modified NewcastleOttawa Scale (NOS) (Table S1) [14]. Studies with scores of 7 points or higher were considered to be of high quality.

\section{Statistical analysis}

The odds ratio (OR) and $95 \%$ confidence interval $(95 \% \mathrm{CI})$ were calculated to investigate the effect strength of the associations between ACE I/D, AGT T704C, AT1R A1166C polymorphisms, and preeclampsia risk. The following genetic models were used: allelic genetic model (ACE I/D: D VS I; AGT T704C: C VS T; AT1R A1166C: C VS A), dominant genetic model (ACE I/D: DD + DI VS II; AGT T704C: CC + CT VS TT; AT1R A1166C: CC + CA VS AA), recessive genetic model (ACE I/D: DD VS DI + II; AGT T704C: CC VS CT + TT; AT1R A1166C: CC VS CA + AA), heterozygote genetic model (ACE I/D: DI VS II; AGT T704C: CT VS TT; AT1R A1166C: CA VS AA), and homozygote genetic model (ACE I/D: DD VS II; AGT T704C: CC VS TT; AT1R A1166C: CC VS AA). The Hardy-Weinberg equilibrium was assessed by the chi-squared test for every study in the control group. Heterogeneity in the meta-analysis was determined by the Cochrane's Q-statistic test, and the inconsistency was quantified with the $\mathrm{I}^{2}$ statistic $\left(\mathrm{I}^{2}\right.$ value more than $50 \%$ or $P$ value less than 0.10 was considered significant heterogeneity and the random effect model was used, otherwise, the fixed-effect model was used). Sensitivity analysis was performed by omitting one study at a time to assess the influence 

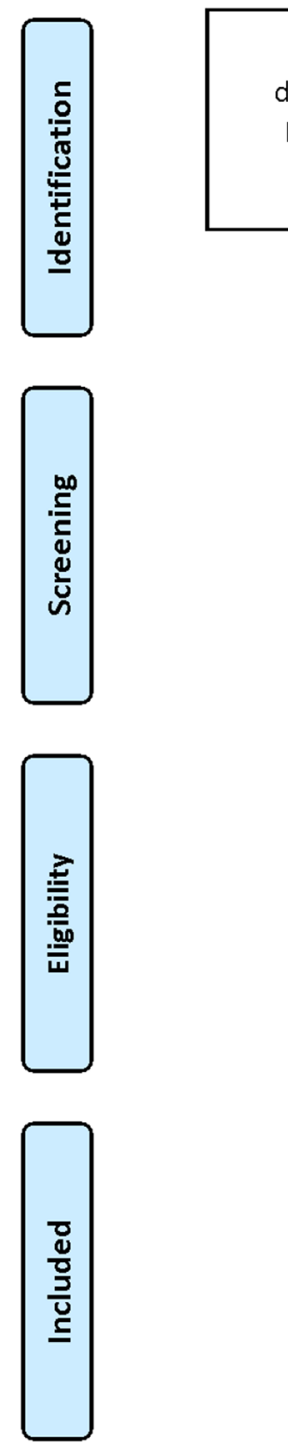

Records identified through database searching PubMed, Embase and Google scholar $(n=280)$
Fig. 1 PRISMA 2009 flow diagram

of each study on the pooled results. Subgroup analysis was conducted, stratifying by geography (Asian, Europe, Africa, America and Australia), ethnicity (Caucasoid, Mongoloid, Black, Mixed race), gestational week (early-onset, late-onset, mixed), PE degree (severe, mild, not mentioned), and patient sample size (less than 100, between 100 and 200, more than 200). Publication bias was evaluated by a visual inspection of funnel plot and Egger's test [15]. If publication bias existed, the "trim and fill" method was used; this method conservatively imputes hypothetical negative unpublished studies to mirror the positive studies that cause funnel plot asymmetry to further assess the possible effect of publication bias [16, 17]. All analyses were performed by Review Manager 5.3 and STATA 12.0 software packages and $P<0.5$ was considered statistically significant.

Trial sequential analysis TSA (trial sequential analysis) (The Copenhagen Trial Unit, Center for Clinical Intervention
Research, Denmark) is a methodology that combines an information size calculation (accumulated sample sizes of all included trials) to reduce type I error and type II error for a meta-analysis with the threshold of statistical significance (http://www.ctu.dk/tsa). TSA was introduced into our metaanalysis. The required information size was calculated based on an overall type I error of 5\%, a power of $90 \%$, and a relative risk reduction (RRR) assumption of $10 \%$.

\section{Results}

\section{The characteristics of eligible studies}

Table 1 and Fig. 1 show the main characteristics of the included studies and the study selection flow chart, respectively. A total of forty studies were finally included in our metaanalysis $[1,5,7-9,18-52]$, among which thirty-four studies 


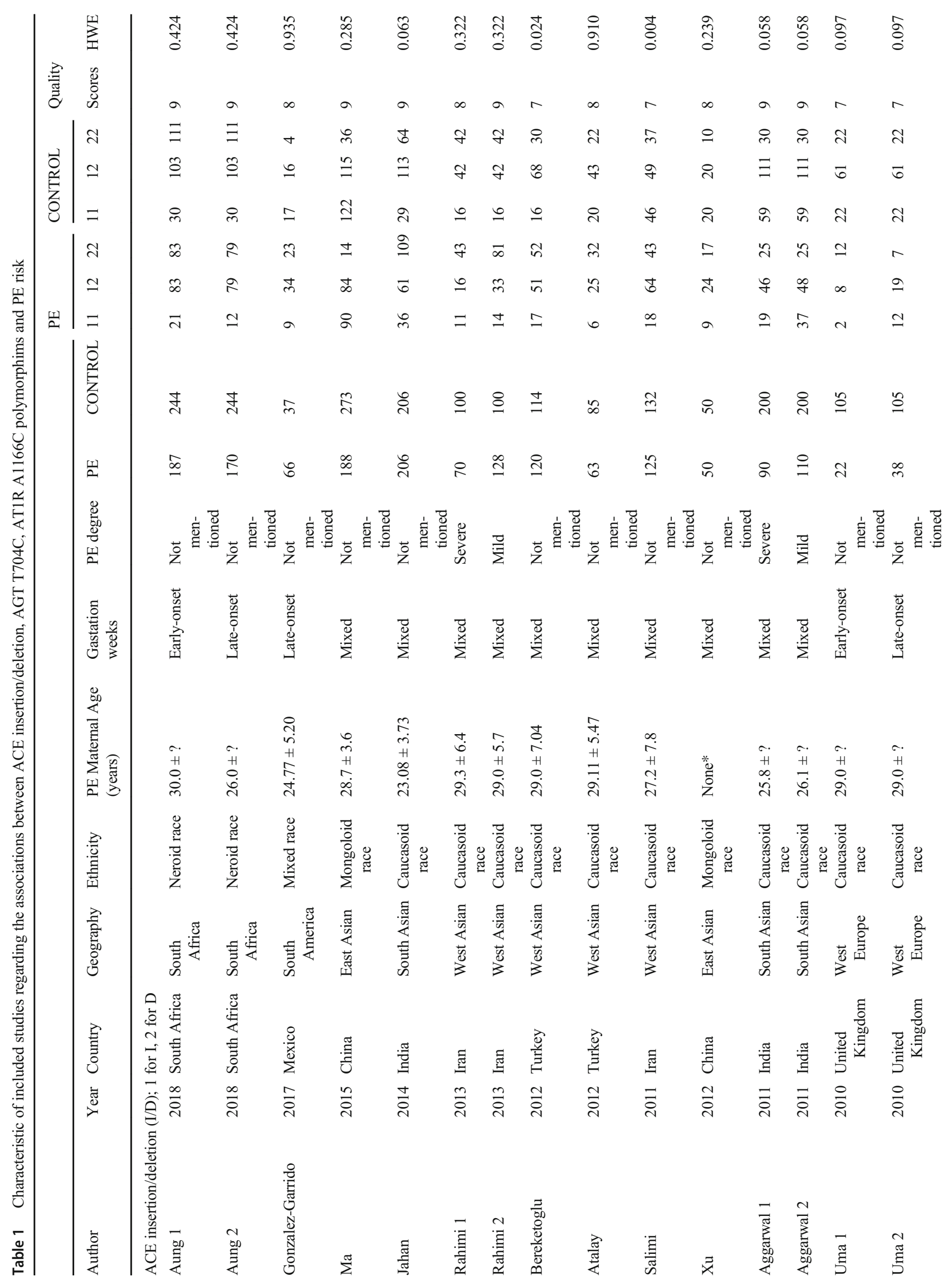




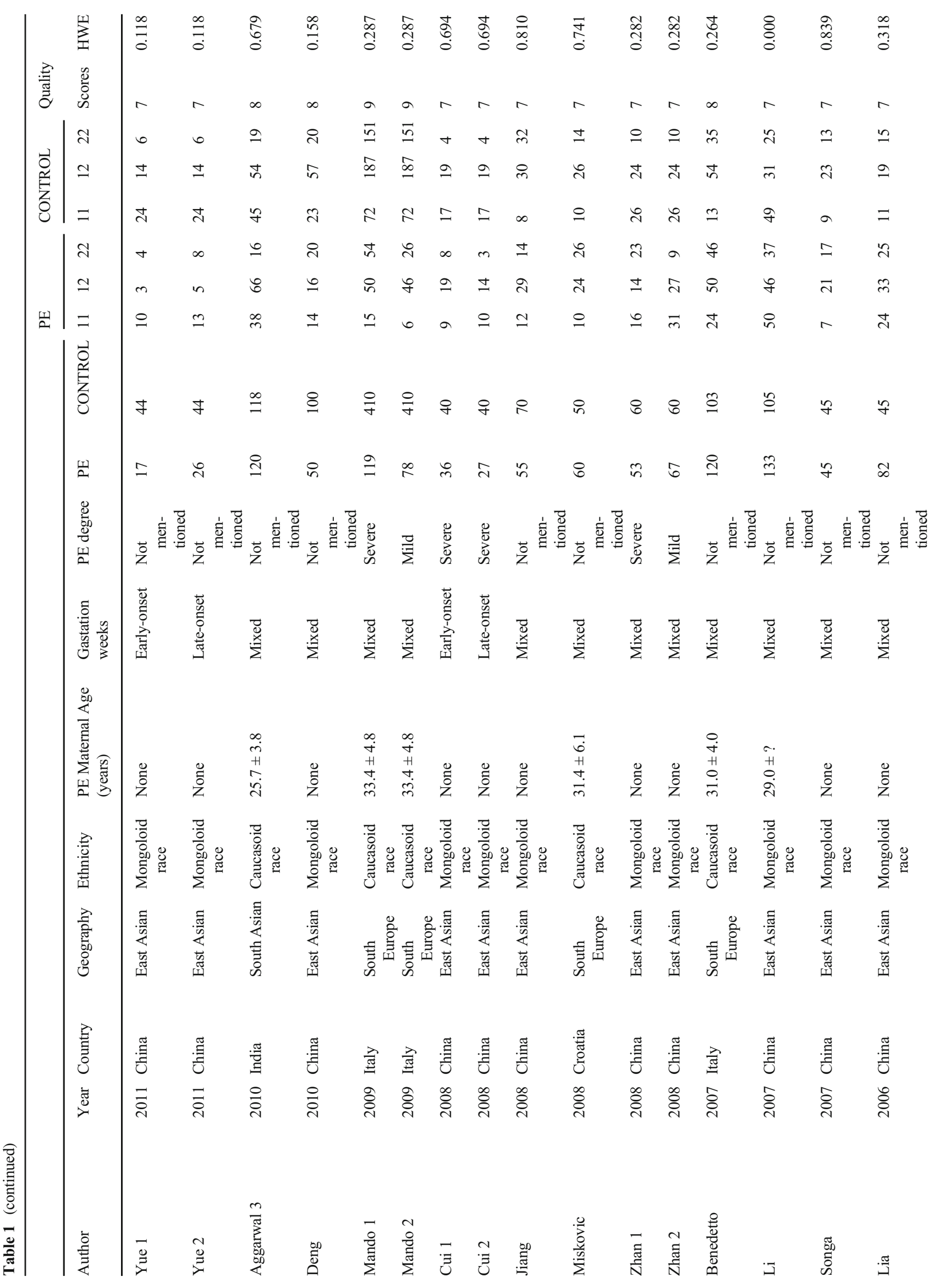




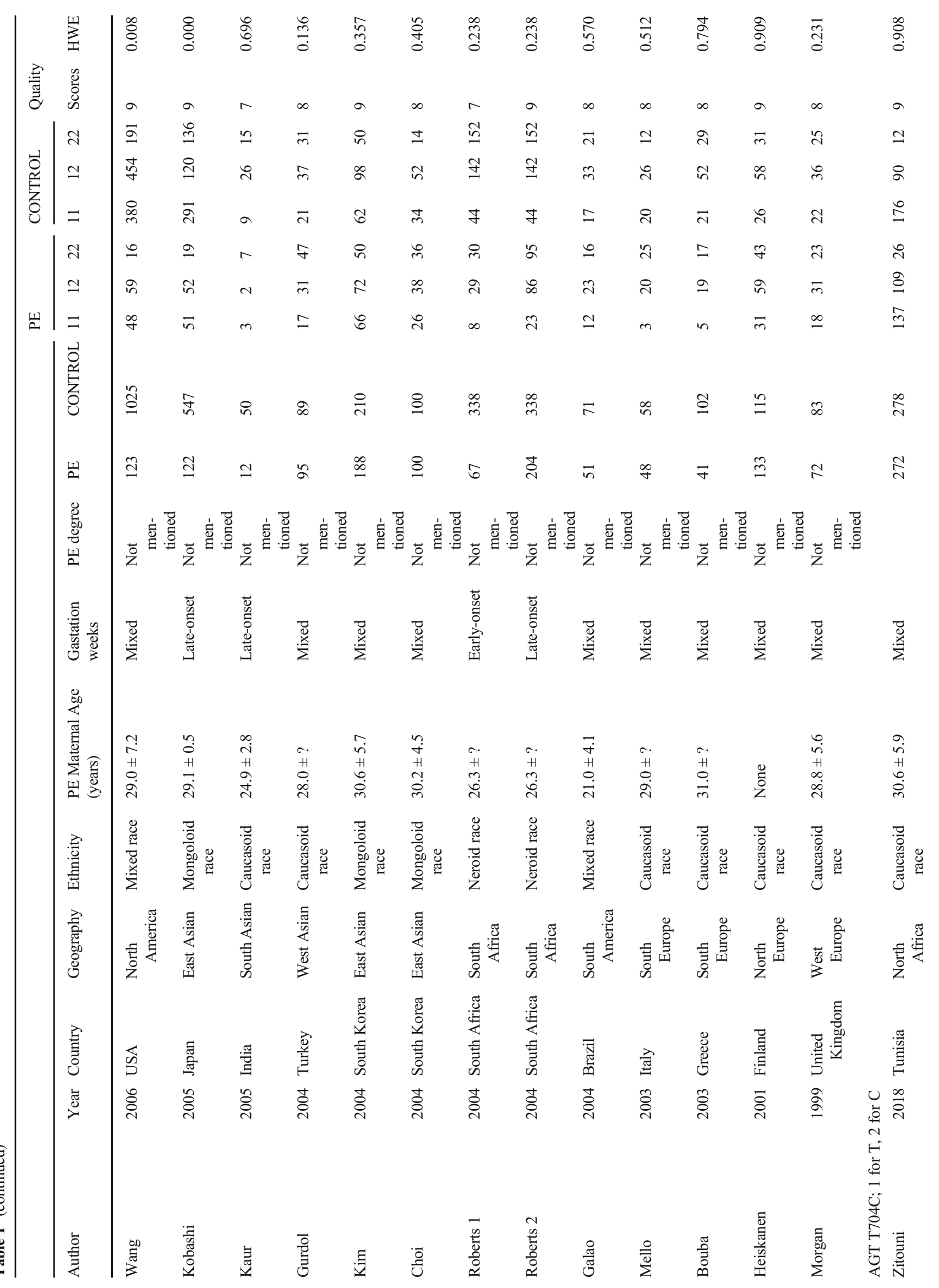




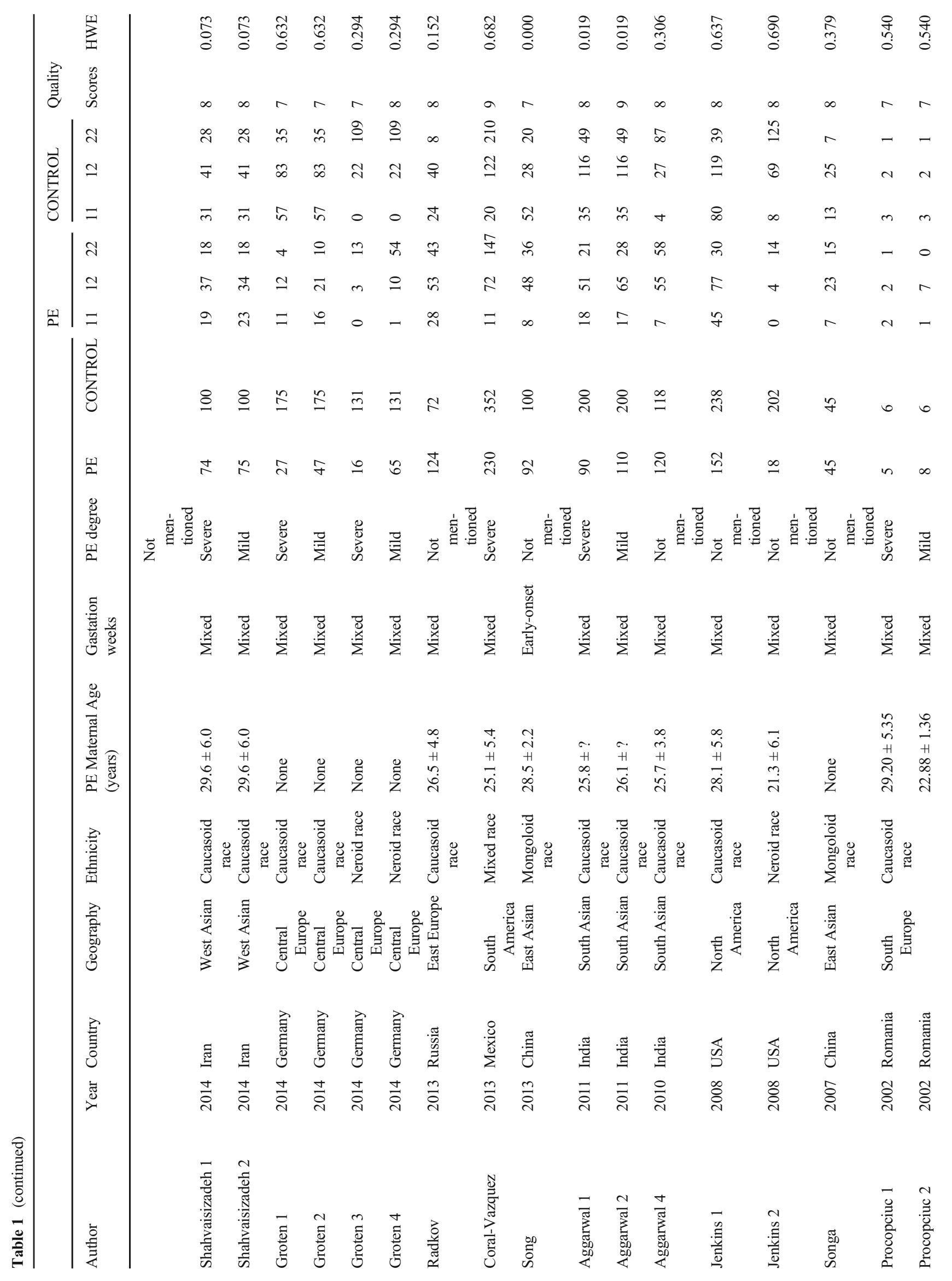




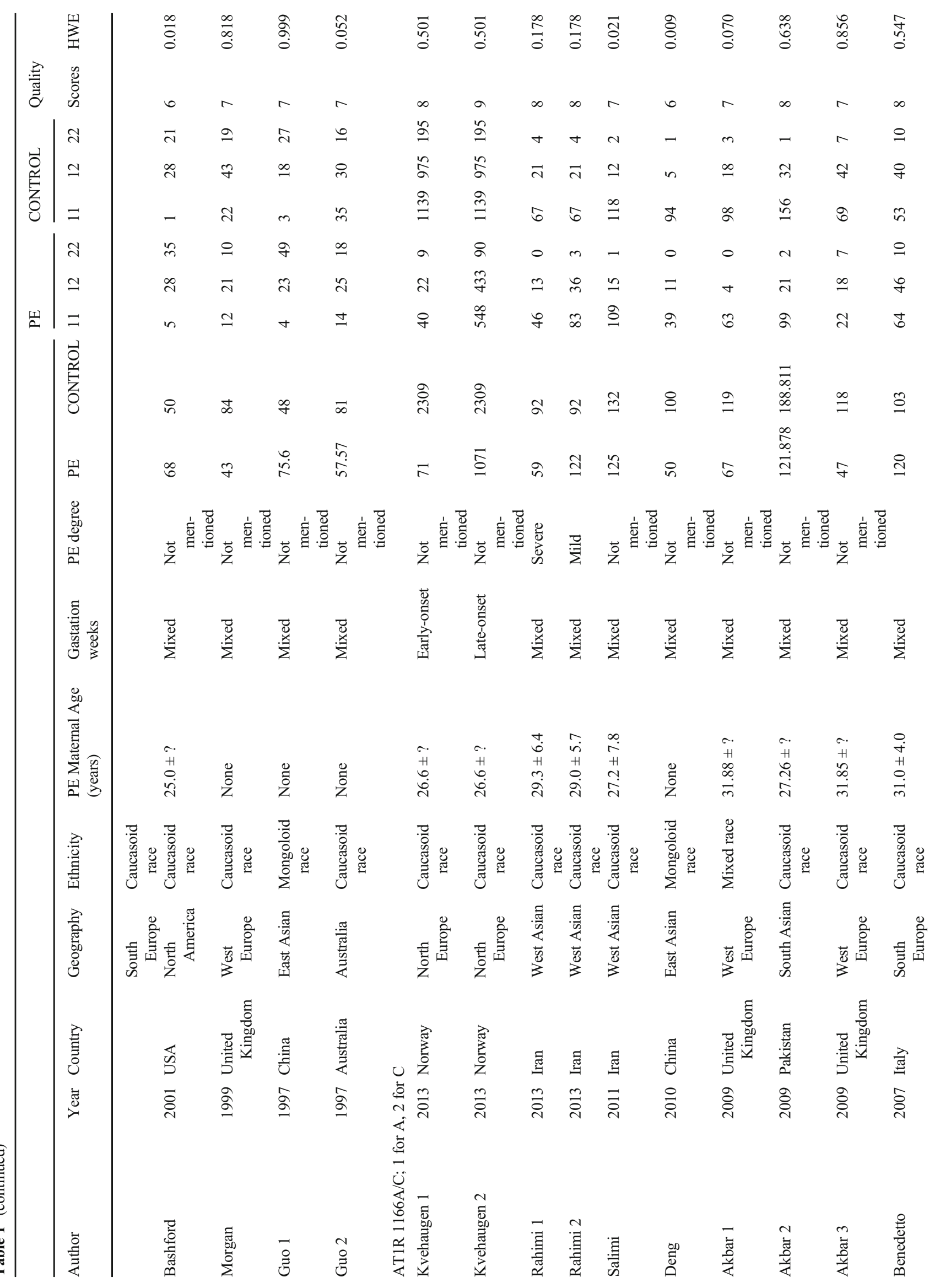


J Assist Reprod Genet (2020) 37:3121-3142

3129

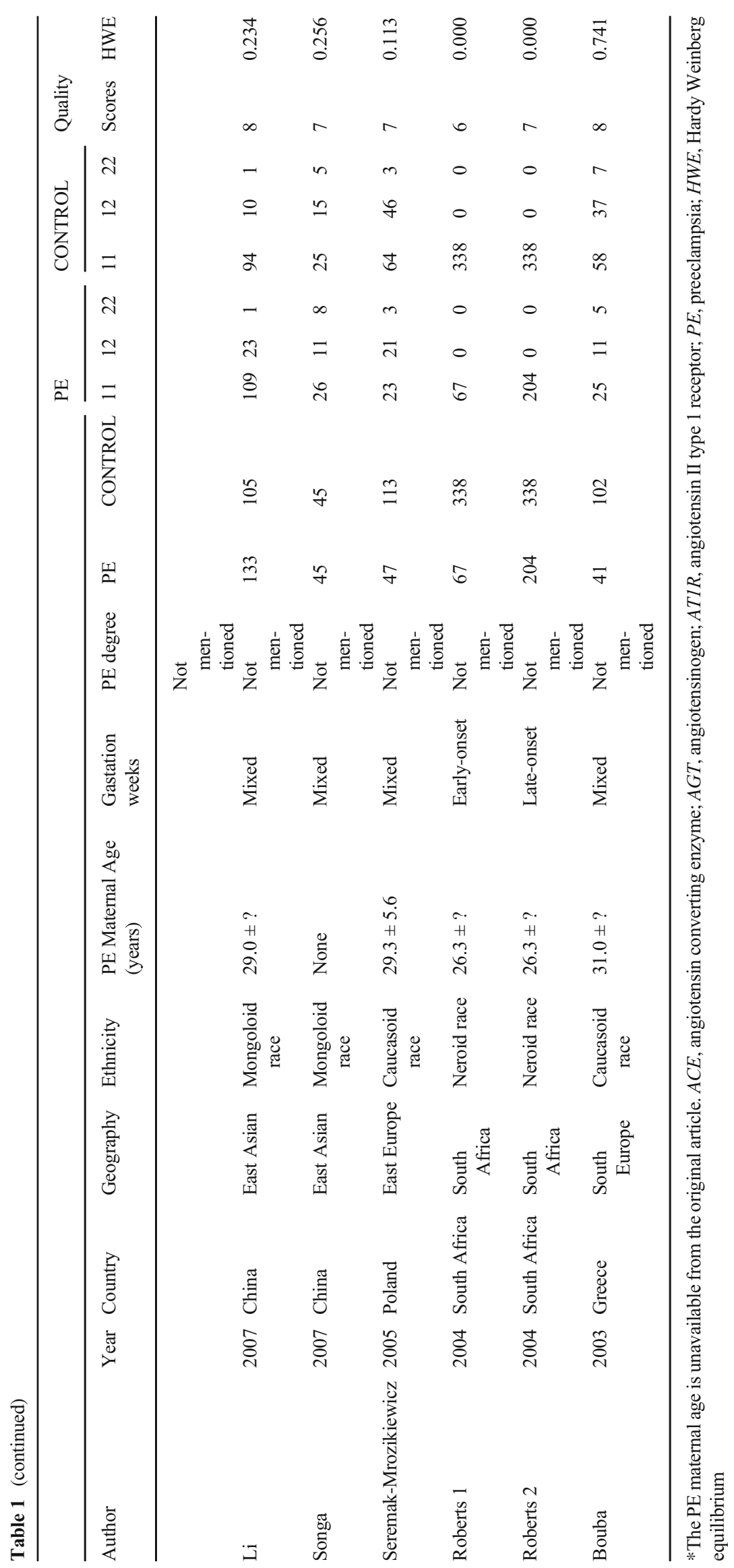

Springer 
involving 3977 patients and 7065 controls regarded the ACE I/D polymorphism, eighteen studies involving 1814 patients and 2892 controls regarded associations with AGT T704C polymorphism, and twelve studies involving 2391 cases and 6604 controls regarded the AT1R A1166C polymorphism.

\section{Meta-analysis results}

Table 2 summarizes the overall and subgroup results regarding the associations between the ACE I/D, AGT T704C, and AT1R A1166C polymorphisms and PE risk. Extensive significant associations were observed for ACE I/D and AGT T704C polymorphisms; however, for the AT1R A1166C polymorphism, no association was detected.

\section{AGT T704C polymorphism}

As summarized in Table 2, the overall analysis indicated that the AGT T704C polymorphism was associated with PE risk in three genetic models (dominant genetic model: $\mathrm{CC}+\mathrm{CT} \mathrm{VS}$ TT: $\mathrm{OR}=1.33,95 \% \mathrm{CI}=1.12-1.59$ (Fig. 3); heterozygote genetic model: $\mathrm{OR}=1.26,95 \% \mathrm{CI}=1.05-1.52$ : homozygote genetic model: $\mathrm{OR}=1.44,95 \% \mathrm{CI}=1.14-1.83$ ). No heterogeneity was observed in the three genetic models. For subgroup analysis by geography, no significant association was detected (Fig. 4b). As stratified by ethnicity, the AGT T704C polymorphism was associated with PE risk both in Caucasoid and Mongoloid populations (Caucasoid: dominant genetic model: $\mathrm{CC}+\mathrm{CT}$ VS TT: $\mathrm{OR}=1.30,95 \% \mathrm{CI}=1.05-1.60$ (Fig. 5b); heterozygote genetic model: CT VS TT: OR = $1.28,95 \% \mathrm{CI}=1.05-1.56$. Mongoloid: allelic genetic model: C VS T: $\mathrm{OR}=1.60,95 \% \mathrm{CI}=1.04-.44$; recessive genetic model: CCVS CT+TT: OR $=4.43,95 \% \mathrm{CI}=2.57-7.62$ ). No associations were also observed in the severe or the mild subgroup either. In the subgroup analysis by patient sample size, significant associations were detected in the dominant $(\mathrm{CC}+$ CT VS TT: $\mathrm{OR}=1.60,95 \% \mathrm{CI}=1.18-2.19)$, recessive $(\mathrm{CC}$ VS CT+TT: $\mathrm{OR}=2.01,95 \% \mathrm{CI}=1.50-2.71)$, and heterozygote $(\mathrm{CT}$ VS TT: $\mathrm{OR}=1.46,95 \% \mathrm{CI}=1.05-2.02)$ genetic model in more than 200 subgroups.

\section{ACE I/D polymorphism}

In the overall analysis, significant associations with significant heterogeneity were observed in the allelic genetic model (D VS I: $\mathrm{OR}=1.29,95 \% \mathrm{CI}=1.16-1.44)$, the dominant genetic model (DD+DI VS II: OR $=1.17,95 \% \mathrm{CI}=1.05-1.31)$, the recessive genetic model (DD VS DI+II: $\mathrm{OR}=1.52,95 \% \mathrm{CI}=$ 1.18-1.94), and the homozygote genetic model (DD VS II: $\mathrm{OR}=1.55,95 \% \mathrm{CI}=1.26-1.91)$ (Fig. 2). Galbraith plot analyses were performed to further explore the sources of heterogeneity, and the figure showed that the studies performed by Mello et al. [46], Gonzalez et al. [1], Choi et al. [45], Atalay et al. [26], Zhan1 et al. [32], Jiang et al. [34], and Ma et al. [18] primarily contributed to the heterogeneity. After excluding these studies, the heterogeneity decreased significantly (I2 = $21 \%$ and PHeterogeneity $=0.14$ for D VS I; I2 $=6 \%$ and PHeterogeneity $=0.37$ for DD+DI VS II; $\mathrm{I} 2=14 \%$ and PHeterogeneity $=0.25$ for DD VS DI + II; $\mathrm{I} 2=0$ and PHeterogeneity $=0.58$ for DD VS II). For subgroup analysis stratified by geography, the ACE ID polymorphism was similarly associated with PE risk in three genetic models in the Asian population (allelic genetic model: D VS I: OR $=1.31$, $95 \% \mathrm{CI}=1.13-1.53$; recessive genetic model: DD VS DI+II: $\mathrm{OR}=1.80,95 \% \mathrm{CI}=1.33-2.43$; homozygote genetic model: DD VS II: OR $=1.53,95 \% \mathrm{CI}=1.16-2.01$ (Fig. 4a)) Regarding the ethnicity subgroup analysis, significant associations were only observed in allelic (D VS I: OR $=1.39$, $95 \% \mathrm{CI}=1.21-1.60)$ and homozygote genetic models (DD VS II: OR $=1.68,95 \% \mathrm{CI}=1.30-2.17$ ) in Caucasoid. However, for the subgroup analysis of gestational weeks, no significant association was detected in both early-onset and late-onset subgroups. In the severe PE subgroup, the ACE I/ D polymorphism was associated with $\mathrm{PE}$ in allelic genetic (D VS I: OR $=1.53,95 \% \mathrm{CI}=1.28-1.83)$, dominant (DD+DI VS II: $\mathrm{OR}=1.50,95 \% \mathrm{CI}=1.11-2.04$ ), and homozygote (DD VS II: $\mathrm{OR}=2.14,95 \% \mathrm{CI}=1.49-3.09)$ genetic models. For the subgroup of patient sample size less than 100 , wide associations with PE risk were observed in allelic (D VS I: $\mathrm{OR}=1.41$, $95 \% \mathrm{CI}=1.19-1.66)$, dominant (DD+DI VS II: OR $=1.37$, $95 \% \mathrm{CI}=1.09-1.73)$, recessive $(\mathrm{DD}$ VS DI+II: OR $=1.50$, $95 \% \mathrm{CI}=1.05-2.15$ ), and homozygote (DD VS II: $\mathrm{OR}=1.85$, $95 \% \mathrm{CI}=1.37-2.51$ (Fig. $5 \mathrm{a})$ ) genetic models..

\section{AT1R A1166C polymorphism}

As shown in Table 2, significant associations were observed in mixed race, early-onset, late-onset, and more than 200 subgroups; however, only one study was analyzed in these subgroups and the results required interpretation with caution (Figs. 4 and 5).

\section{Sensitivity analysis and publication bias}

Sensitivity analysis was performed, and every study was omitted one a time, without any effect on our overall statistical results, indicating that the results were stable and reliable (Fig. 6). Begg's and Egger's test were conducted to analyze publication bias ( $P=0.015$ for ACE I/D polymorphism; $P=$ 0.627 for AGT T704C polymorphism) (Fig. 7). Our results indicated that publication bias was existed in ACE I/D polymorphism; therefore, we applied a sensitivity analysis using the trim and fill method [16], which conservatively imputed hypothetical negative unpublished studies to mirror the positive studies that cause funnel plot asymmetry; the imputed studies of ACE I/D polymorphism produced a symmetrical 


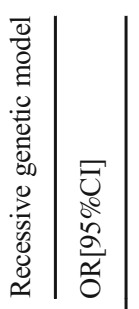

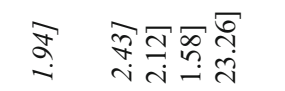

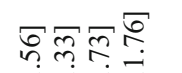

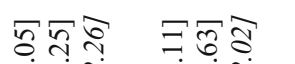

돈동

F

लृ

주웜 $\bar{\sigma}$

कें केष

于ें

कंते

s.

तो तm

$\Rightarrow$ m

तิ่

¿

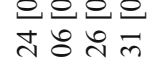

은

은든

의의

क्षें

उ़ि के

응

竞:

o o o 능

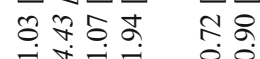

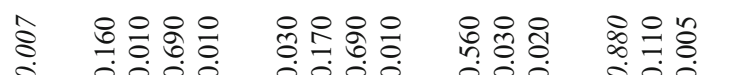

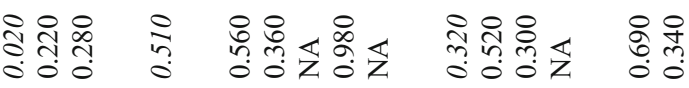

\#

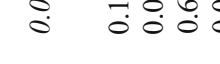

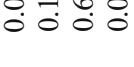

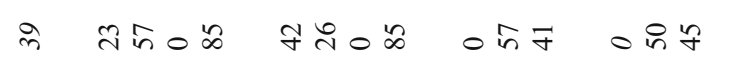

สำ

- $ᄋ$ 艺。苂

ア๐r $\mathbb{Z}$ ○ᄋ

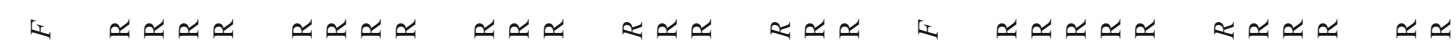

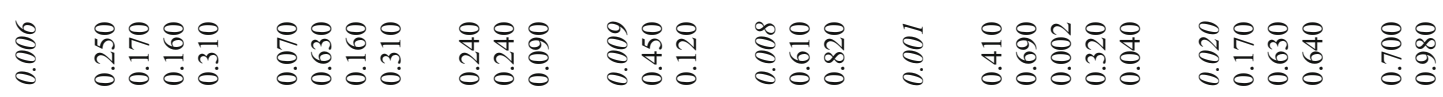

$7=75$

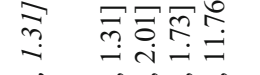

है लूळ की

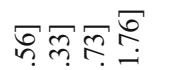

অृत्र

항

.

돈

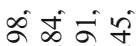

क人

ㅊำ

$\lesssim \quad 0000$

으으응

으의

¿००

จेंक

$\leq 0$

กิ้

水

중윰ำ

-

कले तो है

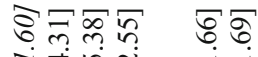

¿ $00 \dot{0}$

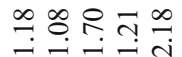

हतेष

¿엥ㅇㅇ

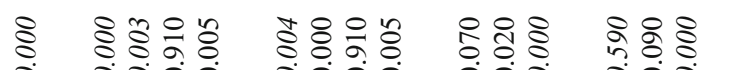

$\therefore \quad 0.0$

0.90

5
5
0

$\stackrel{8}{8}$

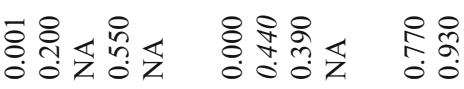

8

$\checkmark n \circ \infty$

กิ

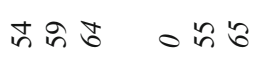

$\sin 8$

8

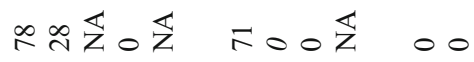

$\approx$

(1)

$\simeq \simeq \approx$

$\approx \simeq \approx$

$\approx \simeq \simeq$

$\simeq$

$\simeq \simeq \simeq \simeq \simeq$

$\simeq \simeq \simeq \simeq \simeq$

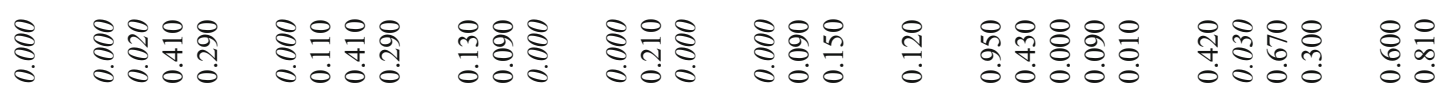

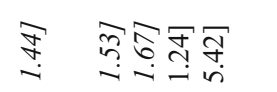

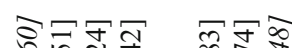

\section{$\bar{m}=\sqrt{2}$}

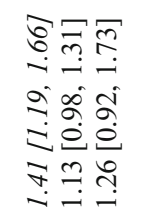

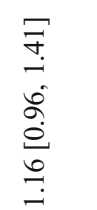

즈

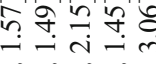

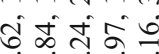

炙名糸 $\bar{m}$

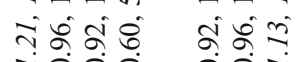

บें

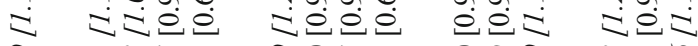

करतिक कर्ष

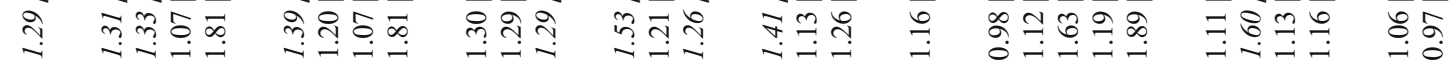

กำ + น

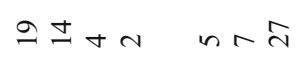

$0+\hat{\mathrm{N}}$

$\stackrel{2}{\sim}=\sim$

$\infty$

INm- no 


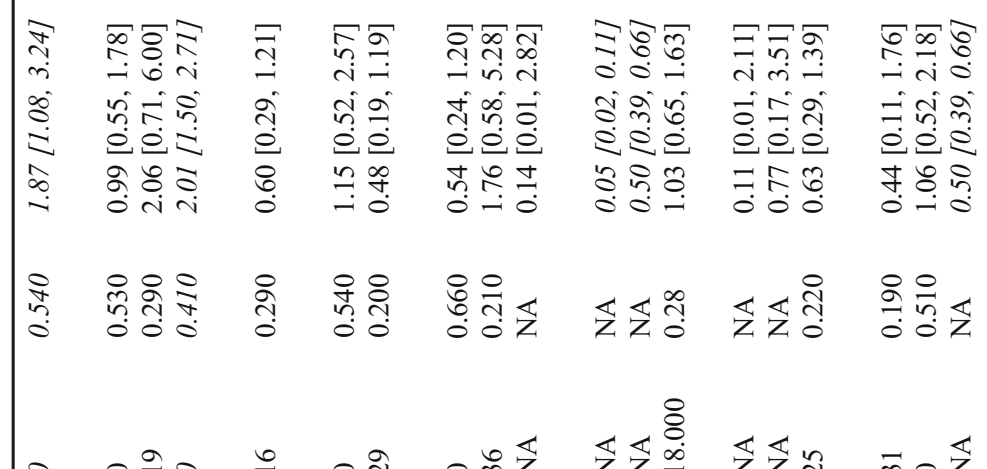

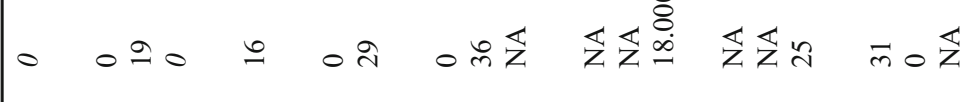

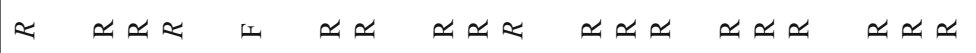

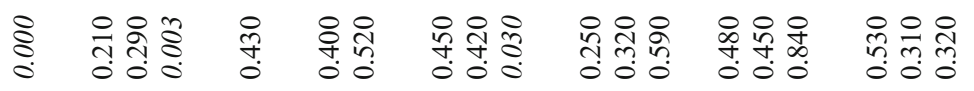

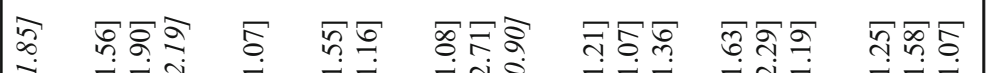

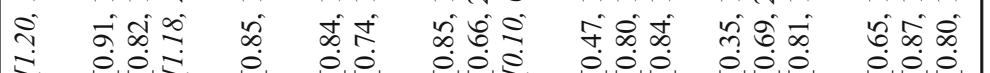

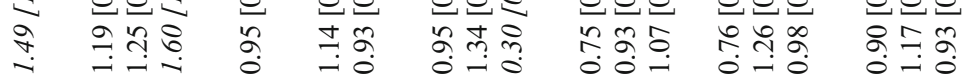

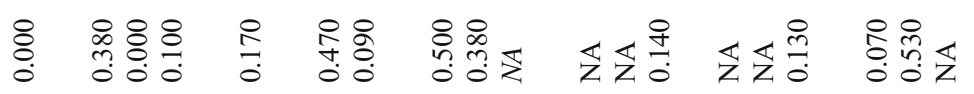

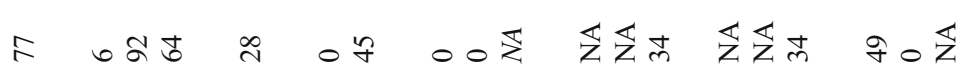

$\simeq \simeq \simeq \simeq$ म $\simeq \simeq \quad \simeq \simeq \approx \simeq \simeq \simeq \simeq \simeq \simeq \simeq$

章各各

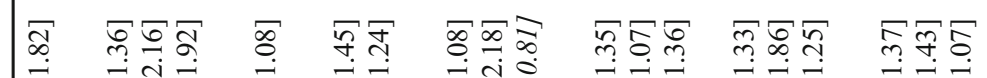

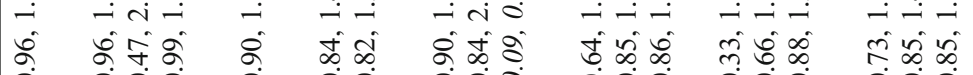

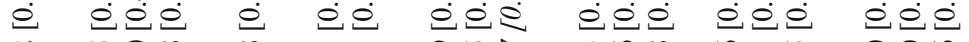

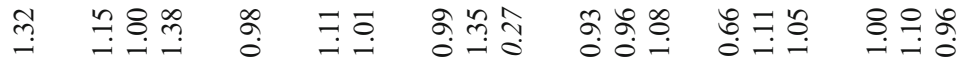

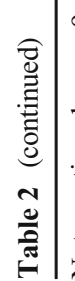

a ma I nn an- h- -

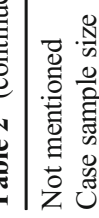

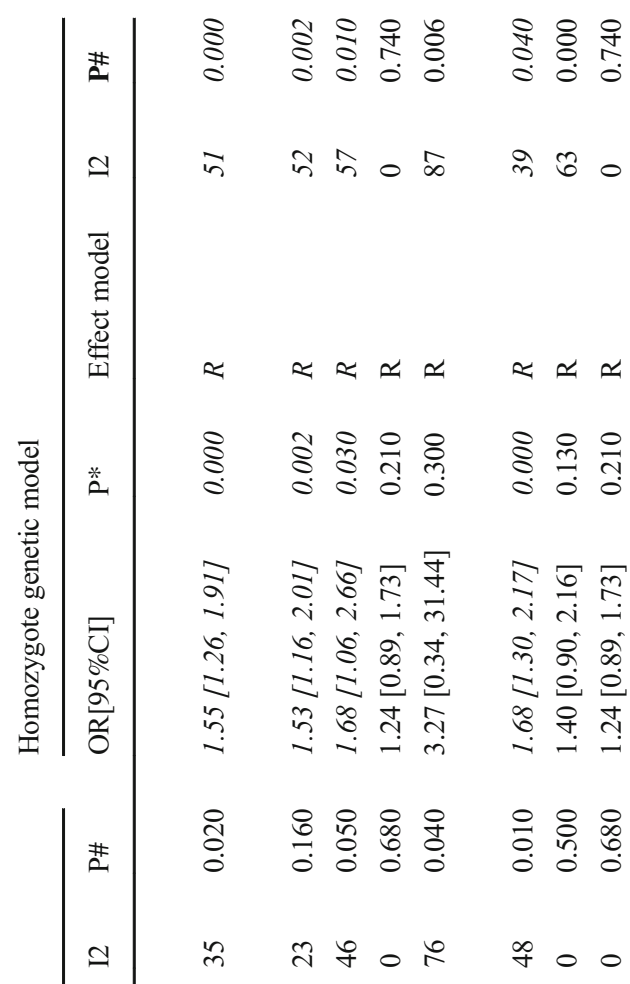

w. $\simeq \simeq \simeq \simeq \simeq \simeq$

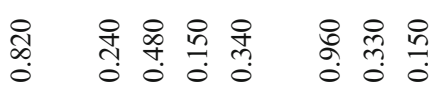

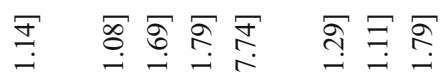

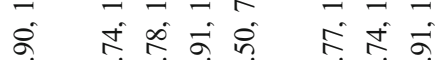

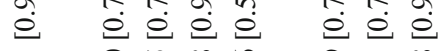

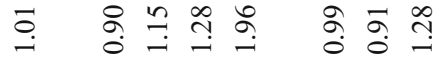

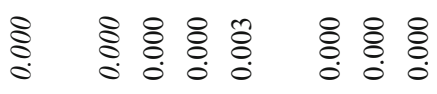

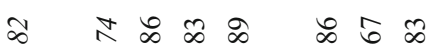

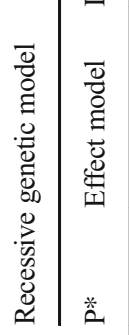

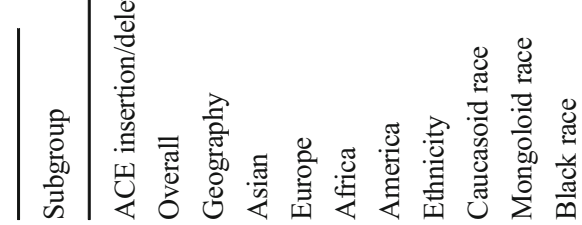




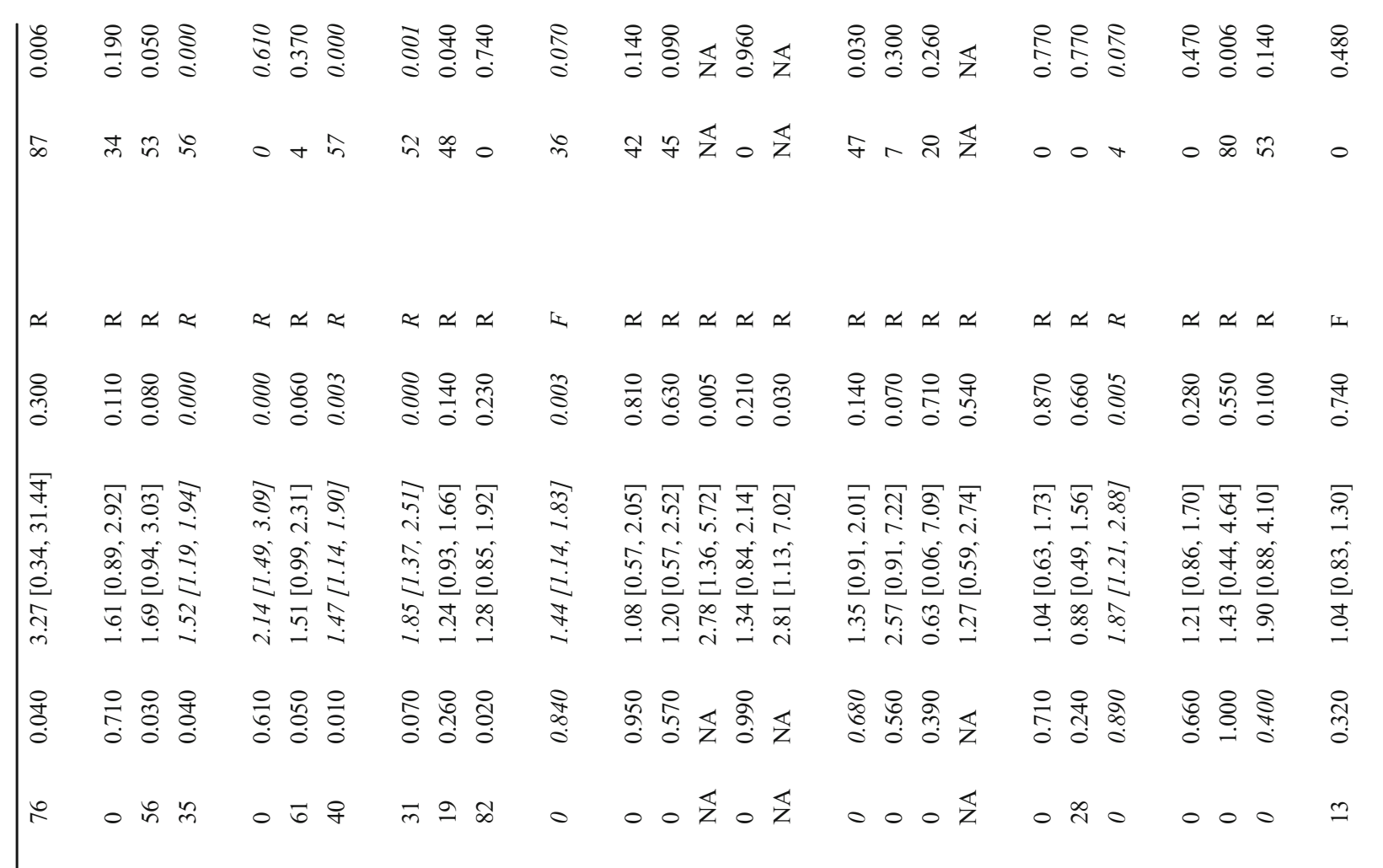

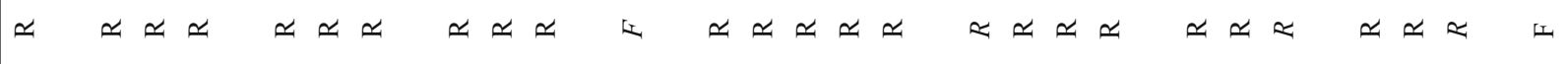

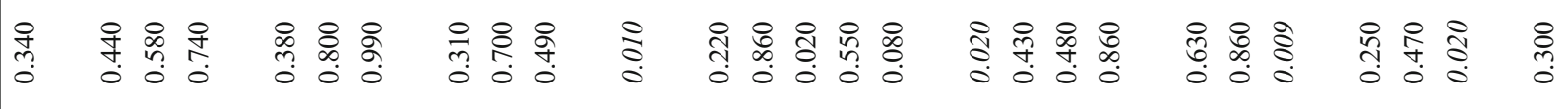

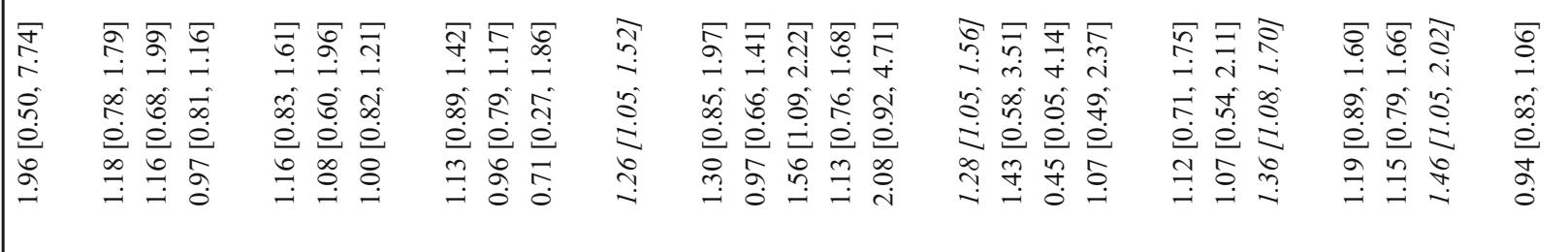

菅

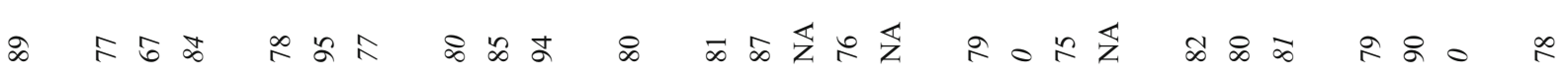

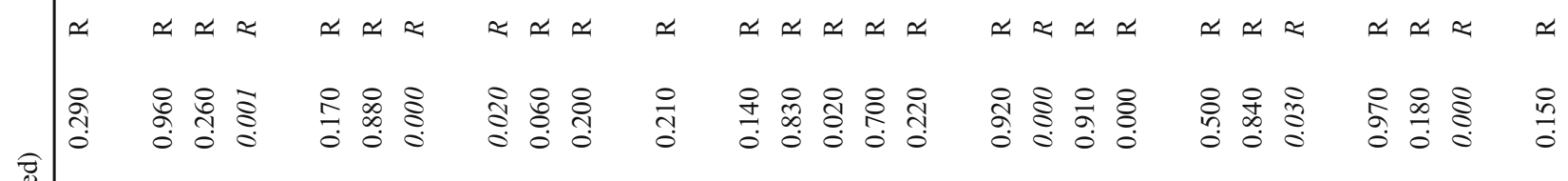

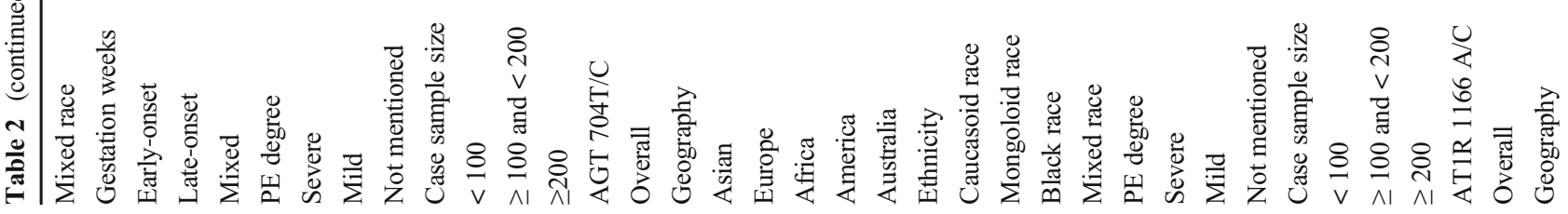




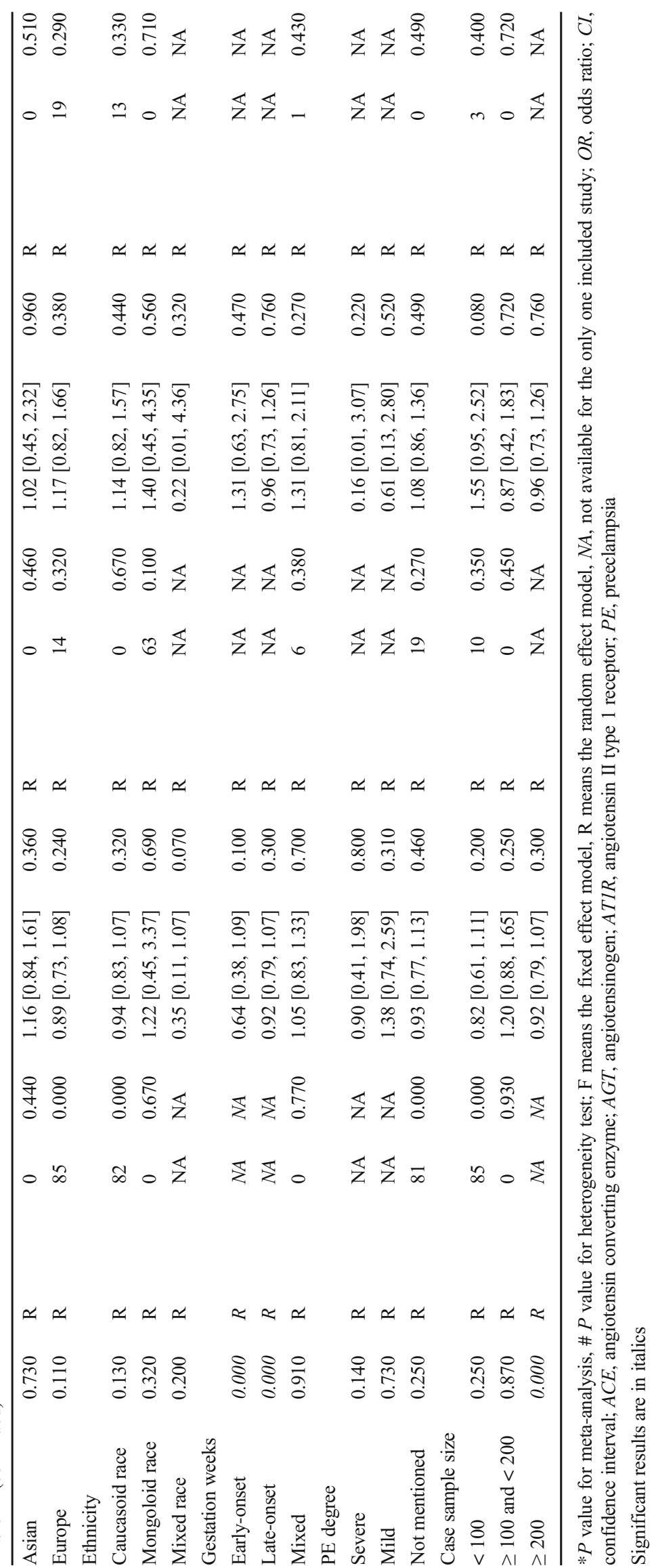




\section{Overall analysis of ACE I/D polymorphism and PE risk}

\begin{tabular}{|c|c|c|c|c|c|c|c|c|c|c|c|}
\hline Study or Subgroup & $\begin{array}{l}\text { PE } \\
\text { Events }\end{array}$ & Total & $\begin{array}{l}\text { Contr } \\
\text { Events }\end{array}$ & Total & Weight & $\begin{array}{c}\text { Odds Ratio } \\
\text { M-H, Random, } 95 \% \mathrm{Cl}\end{array}$ & Year & & $\begin{array}{r}\text { Odds } \\
\text { M-H, Rand }\end{array}$ & $\begin{array}{l}\text { Ratio } \\
\text { lom, } 95 \% \mathrm{Cl}\end{array}$ & \\
\hline Aung 2 & 79 & 91 & 111 & 141 & $3.2 \%$ & $1.78[0.86,3.69]$ & 2018 & & & & \\
\hline Aung 1 & 83 & 104 & 111 & 141 & $3.6 \%$ & $1.07[0.57,2.00]$ & 2018 & & & & \\
\hline Gonzalez-Garrido & 23 & 32 & 4 & 21 & $1.7 \%$ & $10.86[2.86,41.24]$ & 2017 & & & & \\
\hline $\mathrm{Ma}$ & 14 & 104 & 36 & 158 & $3.4 \%$ & $0.53[0.27,1.04]$ & 2015 & & & & \\
\hline Jahan & 109 & 145 & 64 & 93 & $3.8 \%$ & $1.37[0.77,2.45]$ & 2014 & & & & \\
\hline Rahimi 2 & 81 & 95 & 42 & 58 & $3.0 \%$ & $2.20[0.98,4.95]$ & 2013 & & & & \\
\hline Rahimi 1 & 43 & 54 & 42 & 58 & $2.7 \%$ & $1.49[0.62,3.58]$ & 2013 & & & & \\
\hline Atalay & 32 & 38 & 22 & 42 & $2.2 \%$ & $4.85[1.68,14.02]$ & 2012 & & & & \\
\hline$x u$ & 17 & 26 & 10 & 30 & $2.1 \%$ & $3.78[1.25,11.45]$ & 2012 & & & & \\
\hline Aggawal 2 & 25 & 62 & 30 & 89 & $3.4 \%$ & $1.33[0.68,2.60]$ & 2011 & & & & \\
\hline Aggamal 1 & 25 & 44 & 30 & 89 & $3.2 \%$ & $2.59[1.23,5.43]$ & 2011 & & & & \\
\hline Yue 2 & 8 & 21 & 6 & 30 & $1.8 \%$ & $2.46[0.70,8.64]$ & 2011 & & & & \\
\hline Yue 1 & 4 & 14 & 6 & 30 & $1.4 \%$ & $1.60[0.37,6.92]$ & 2011 & & & & \\
\hline Aggarwal 3 & 16 & 54 & 19 & 64 & $3.0 \%$ & $1.00[0.45,2.20]$ & 2010 & & & & \\
\hline Uma 1 & 12 & 14 & 22 & 44 & $1.3 \%$ & $6.00[1.20,30.00]$ & 2010 & & & & \\
\hline Uma 2 & 7 & 19 & 22 & 44 & $2.1 \%$ & $0.58[0.19,1.76]$ & 2010 & & & & \\
\hline Deng & 20 & 34 & 20 & 43 & $2.6 \%$ & $1.64[0.66,4.08]$ & 2010 & & & & \\
\hline Mando 2 & 26 & 32 & 151 & 223 & $2.6 \%$ & $2.07[0.81,5.24]$ & 2009 & & & & \\
\hline Mando 1 & 54 & 69 & 151 & 223 & $3.6 \%$ & $1.72[0.91,3.25]$ & 2009 & & & & \\
\hline Zhan 2 & 9 & 40 & 10 & 36 & $2.3 \%$ & $0.75[0.27,2.14]$ & 2008 & & & & \\
\hline Zhan 1 & 23 & 39 & 10 & 36 & $2.5 \%$ & $3.74[1.42,9.85]$ & 2008 & & & & \\
\hline Miskovic & 26 & 36 & 14 & 24 & $2.1 \%$ & $1.86[0.62,5.53]$ & 2008 & & & & \\
\hline Jiang & 14 & 26 & 32 & 40 & $2.1 \%$ & $0.29[0.10,0.87]$ & 2008 & & & & \\
\hline Cui 2 & 3 & 13 & 4 & 21 & $1.2 \%$ & $1.27[0.24,6.90]$ & 2008 & & & & \\
\hline Cui 1 & 8 & 17 & 4 & 21 & $1.5 \%$ & $3.78[0.89,16.05]$ & 2008 & & & & \\
\hline Benedetto & 46 & 70 & 35 & 49 & $3.0 \%$ & $0.77[0.35,1.69]$ & 2007 & & & & \\
\hline songa & 17 & 24 & 13 & 22 & $1.9 \%$ & $1.68[0.49,5.72]$ & 2007 & & & & \\
\hline Lia & 25 & 49 & 15 & 26 & $2.5 \%$ & $0.76[0.29,1.99]$ & 2006 & & & & \\
\hline Kaur & 7 & 10 & 15 & 24 & $1.3 \%$ & $1.40[0.29,6.83]$ & 2005 & & & & \\
\hline Roberts 2 & 95 & 118 & 152 & 196 & $3.8 \%$ & $1.20[0.68,2.11]$ & 2004 & & & & \\
\hline Roberts 1 & 30 & 38 & 152 & 196 & $2.8 \%$ & $1.09[0.46,2.54]$ & 2004 & & & & \\
\hline Kim & 50 & 116 & 50 & 112 & $4.0 \%$ & $0.94[0.56,1.59]$ & 2004 & & & & \\
\hline Gurdol & 47 & 64 & 31 & 52 & $3.0 \%$ & $1.87[0.86,4.10]$ & 2004 & & & & \\
\hline Galao & 16 & 28 & 21 & 38 & $2.4 \%$ & $1.08[0.40,2.89]$ & 2004 & & & & \\
\hline Choi & 36 & 62 & 14 & 48 & $3.0 \%$ & $3.36[1.51,7.49]$ & 2004 & & & & \\
\hline Bouba & 17 & 22 & 29 & 50 & $2.0 \%$ & $2.46[0.78,7.73]$ & 2003 & & & & \\
\hline Mello & 25 & 28 & 12 & 32 & $1.6 \%$ & $13.89[3.44,56.05]$ & 2003 & & & & \\
\hline Heiskanen & 43 & 74 & 31 & 57 & $3.3 \%$ & $1.16[0.58,2.33]$ & 2001 & & & & \\
\hline Morgan & 23 & 41 & 25 & 47 & $2.8 \%$ & $1.12[0.48,2.61]$ & 1999 & & & & \\
\hline Total $(95 \% \mathrm{Cl})$ & & 1967 & & 2748 & $100.0 \%$ & $1.55[1.26,1.91]$ & & & & & \\
\hline Total events & 1238 & & 1568 & & & & & & & & \\
\hline \multicolumn{8}{|c|}{$\begin{array}{l}\text { Heterogeneity: } \text { Tau }^{2}=0.20 ; \mathrm{Chi}^{2}=77.80, \mathrm{df}=38(\mathrm{P}=0.0001) ;\left.\right|^{2}=51 \% \\
\text { Test for overall effect: } Z=4.18(P=0.0001)\end{array}$} & $\begin{array}{cc}1 & 1 \\
0.1 & 0.2\end{array}$ & $\begin{array}{c}0.5 \\
\text { Protective }\end{array}$ & $\begin{array}{l}1 \\
\text { Risky }\end{array}$ & 10 \\
\hline
\end{tabular}

Fig. 2 Overall analysis of ACE I/D polymorphism and PE risk

funnel plot [53] (Fig. 7a). The shape of funnel plot was symmetrical for the AGT T704C polymorphism (Fig. $7 b$ ), implying that there was no publication bias for this polymorphism.

\section{Trial sequential analysis}

We performed a TSA for the homozygote genetic model of ACE I/D polymorphism and dominant genetic model of AGT T704C polymorphism (Fig. 8). The results of the two polymorphisms showed that the blue line of the cumulative z-curve crossed the TSA monitoring boundary and the cumulative sample size was reached, indicating that no further studies were essential to confirm the associations.

\section{Discussion}

In pregnant women with $\mathrm{PE}$, downregulated renin-angiotensin system (RAS) activity is observed, resulting in increased vascular responsiveness to angiotensin II [4]. The increased plasm levels of angiotensin (AGT) and angiotensin converting enzyme (ACE) in PE subjects lead to the augmentation of angiotensin II [5, 54]; moreover, the pathophysiological effects of angiotensin II are enhanced by the upregulation of angiotensin II type 1 receptor (AT1R) [9], causing the dysregulation of blood pressure. Gene polymorphisms were reported to be associated with the abnormal expression of mRNA and protein $[55,56]$. Our meta-analysis demonstrated that the polymorphisms of AGT T704C and ACE I/D were significantly associated with an increased risk of 


\section{Overall analysis of AGT T704C polymorphism and PE risk}

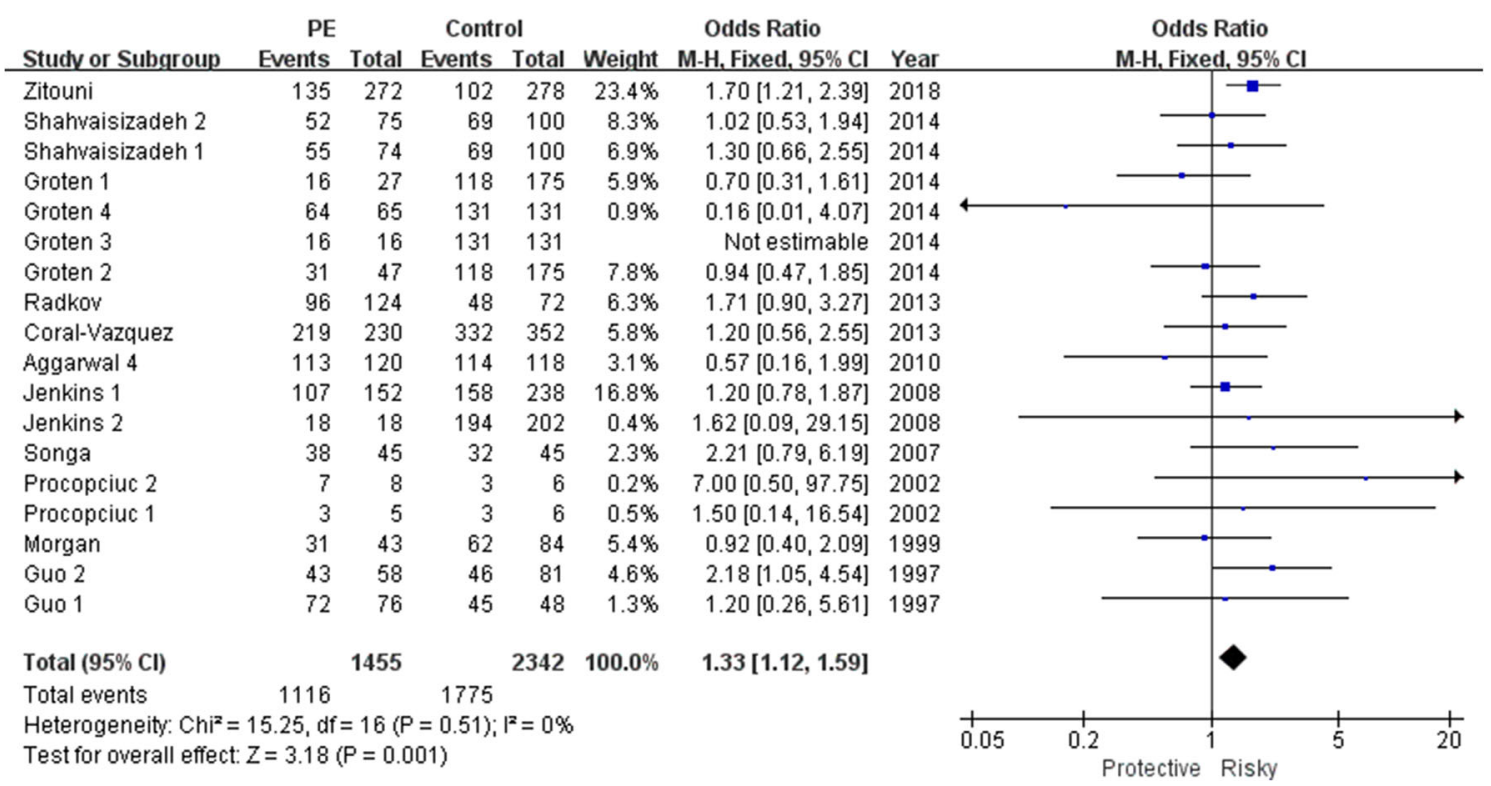

Fig. 3 Overall analysis of AGT T704C polymorphism and PE risk

Subgroup analysis (stratified by Geography) of ACE I/D and AGT T704C polymorphisms and PE risk

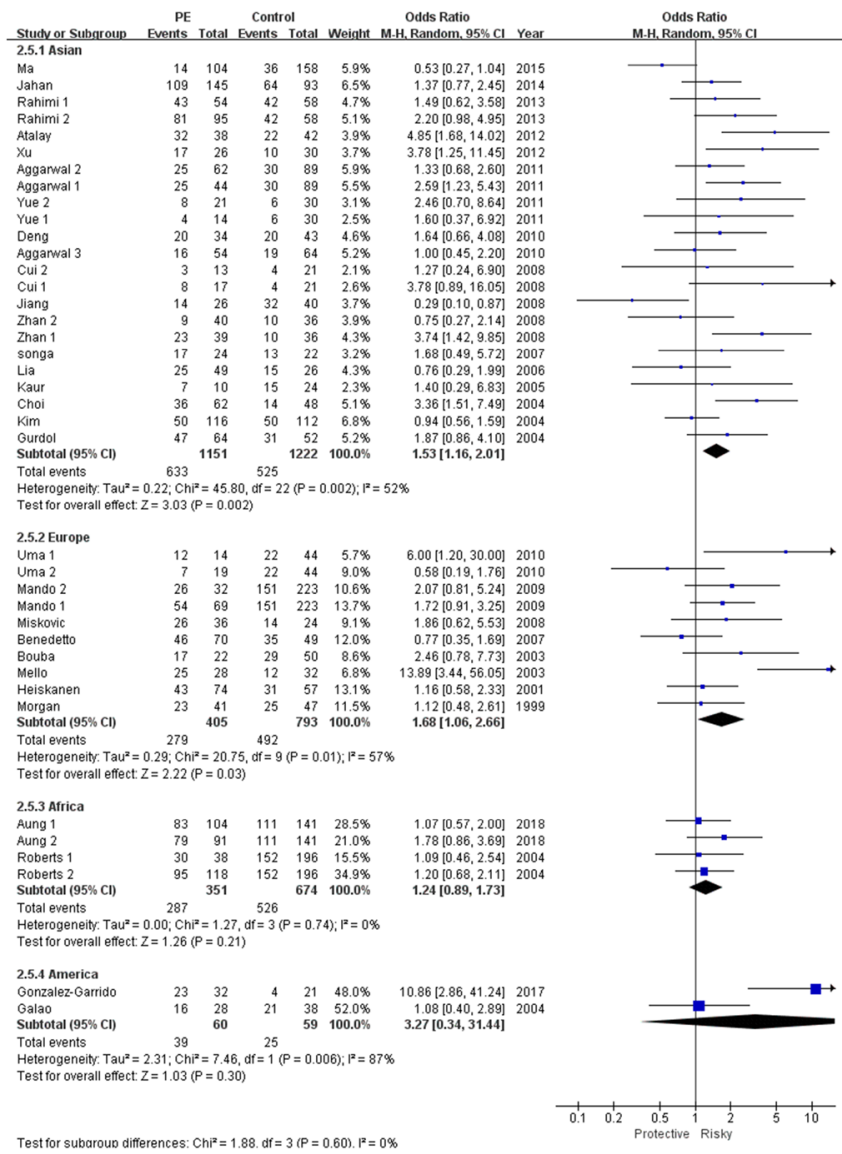

a $\mathrm{ACE} \mathrm{I} / \mathrm{D}$ polymorphism; Homozygote genetic model

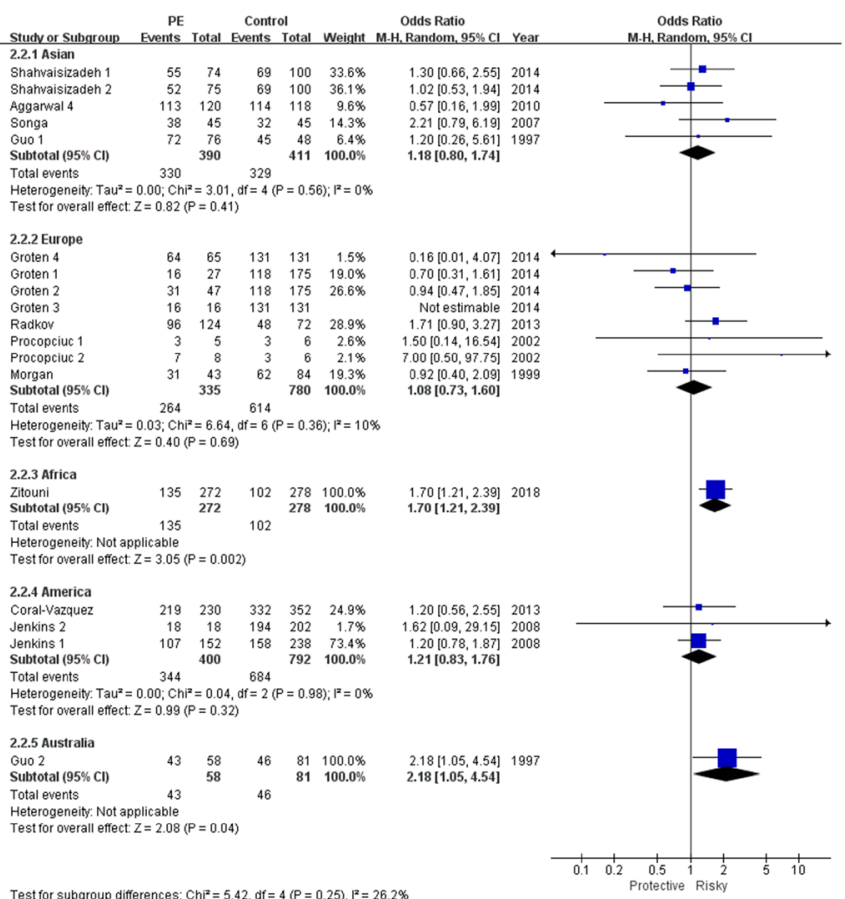

b AGT T704C polymorphism; Dominant genetic model

Fig. 4 Subgroup analysis (stratified by geography) of ACE I/D and AGT T704C polymorphisms and PE risk 
Subgroup analysis (stratified by Ethnicity) of ACE I/D and AGT T704C polymorphisms and PE risk

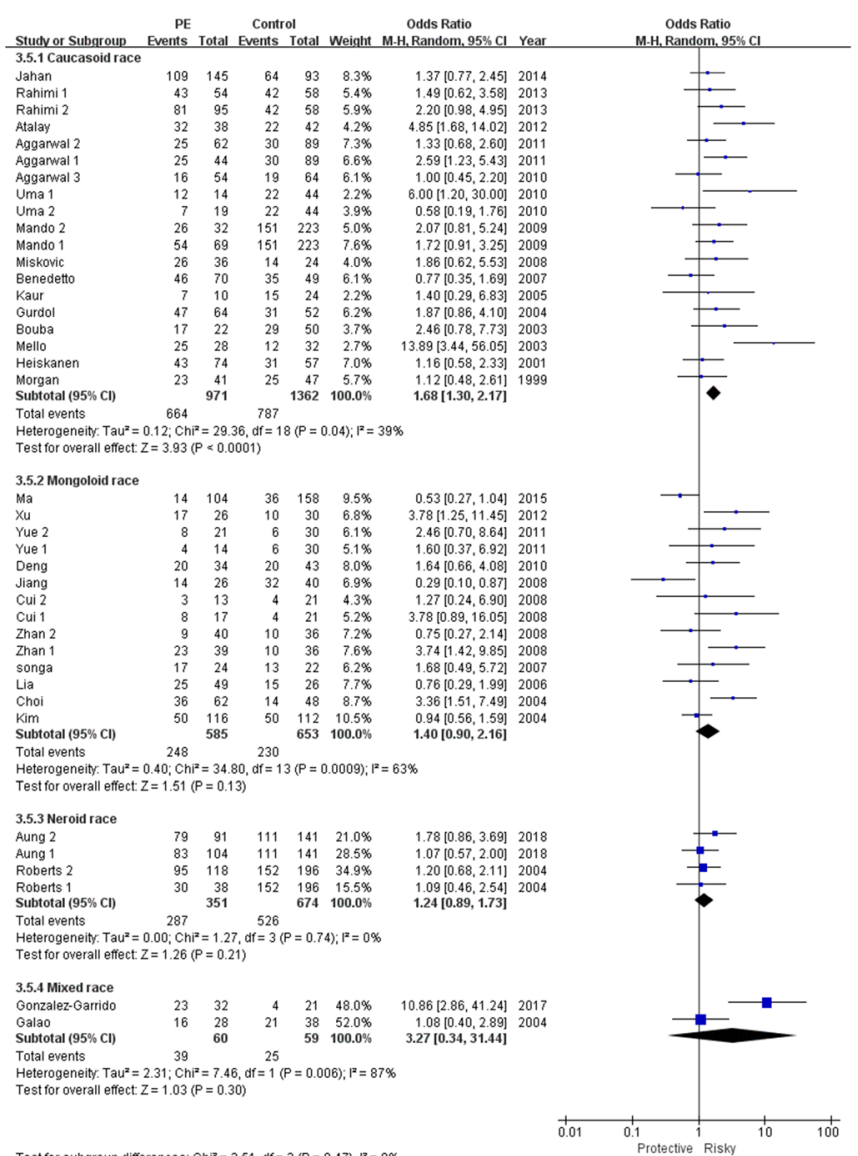

a ACE I/D polymorphism; Homozygote genetic model

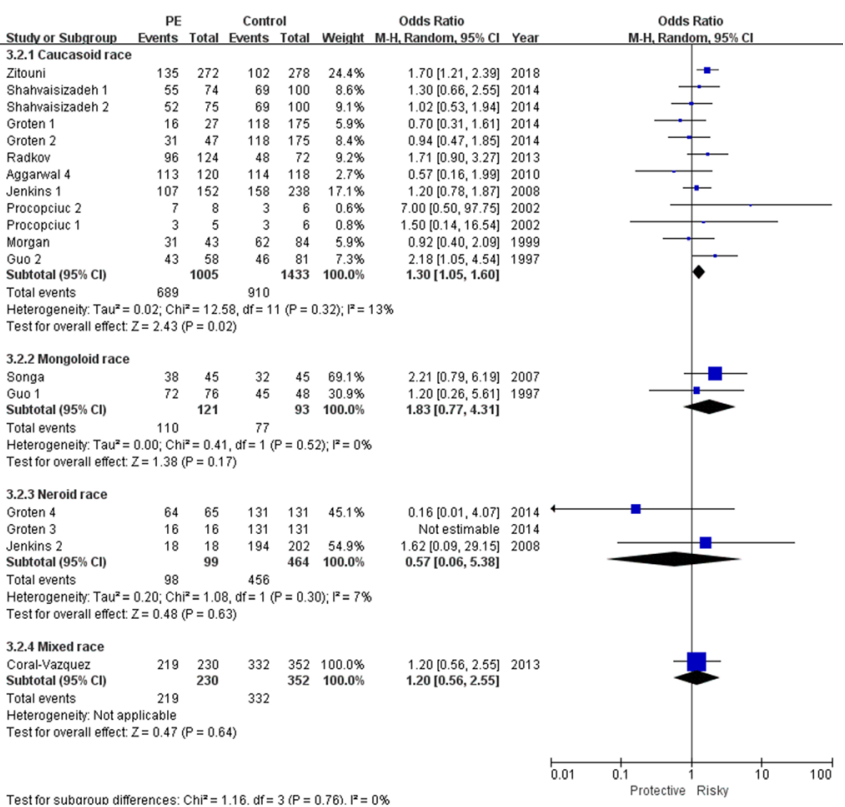

b AGT T704C polymorphism; Dominant genetic model

Fig. 5 Subgroup analysis (stratified by ethnicity) of ACE I/D and AGT T704C polymorphisms and PE risk

preeclampsia $(\mathrm{PE})$ and weak associations of the AT1R A1166C polymorphism with PE were observed.

Previous meta-analyses indicated an increased PE risk with high heterogeneity of ACE I/D and AGT T704C polymorphisms, but no association was observed for the AT1R A1166C polymorphism [57-60]. However, the latest meta-analysis was performed in 2012, and in subsequent years, several studies conducted in different regions and

\section{Sensitivity analysis of ACE I/D and AGT T704C polymorphisms and PE risk}

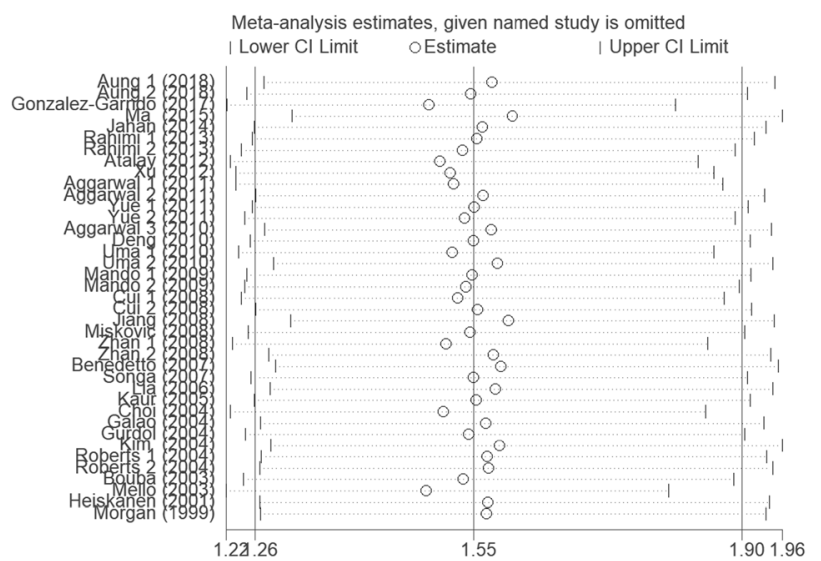

a ACE I/D polymorphism; Homozygote genetic model

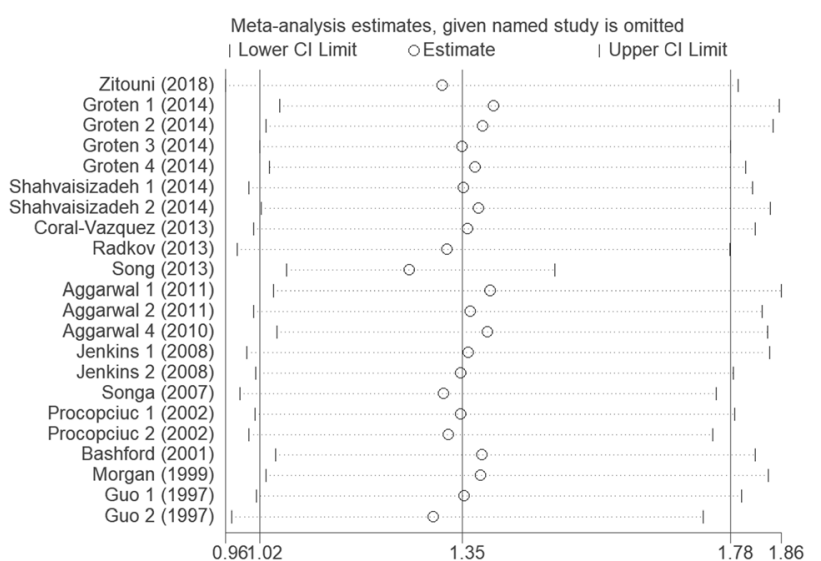

b AGT T704C polymorphism; Dominant genetic model

Fig. 6 Sensitivity analysis of ACE I/D and AGT T704C polymorphisms and PE risk 
Begg' s and filled funnel plot of ACE I/D and AGT T704C polymorphisms and PE risk
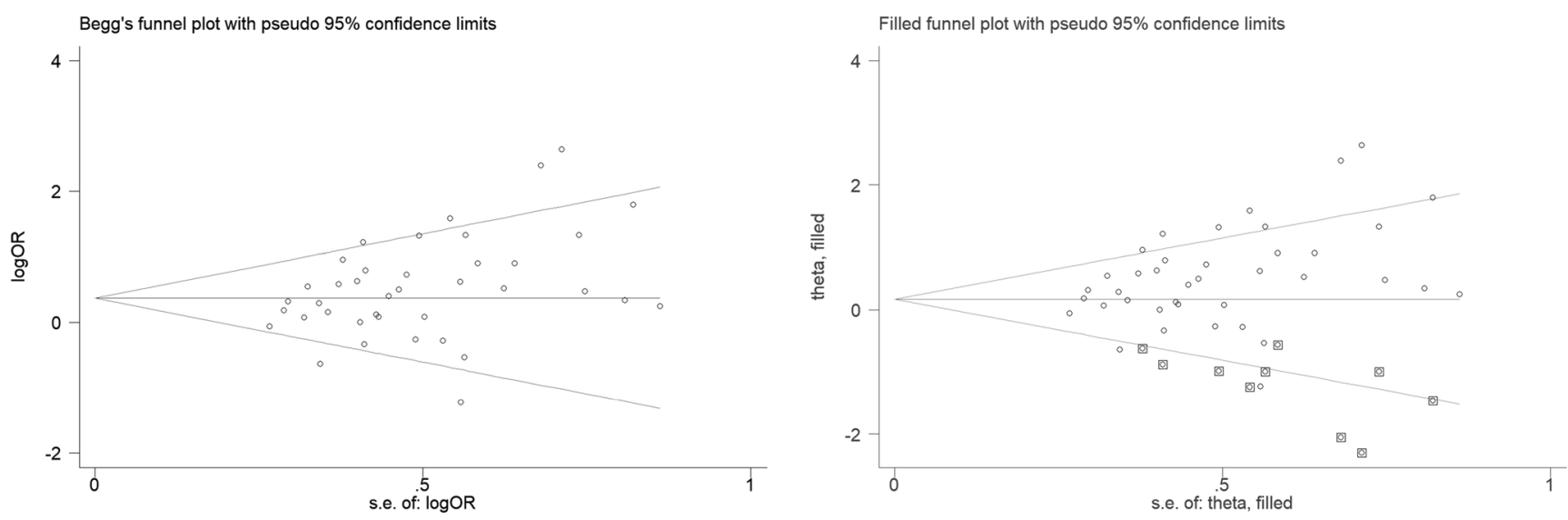

a ACE I/D polymorphism; Homozygote genetic model
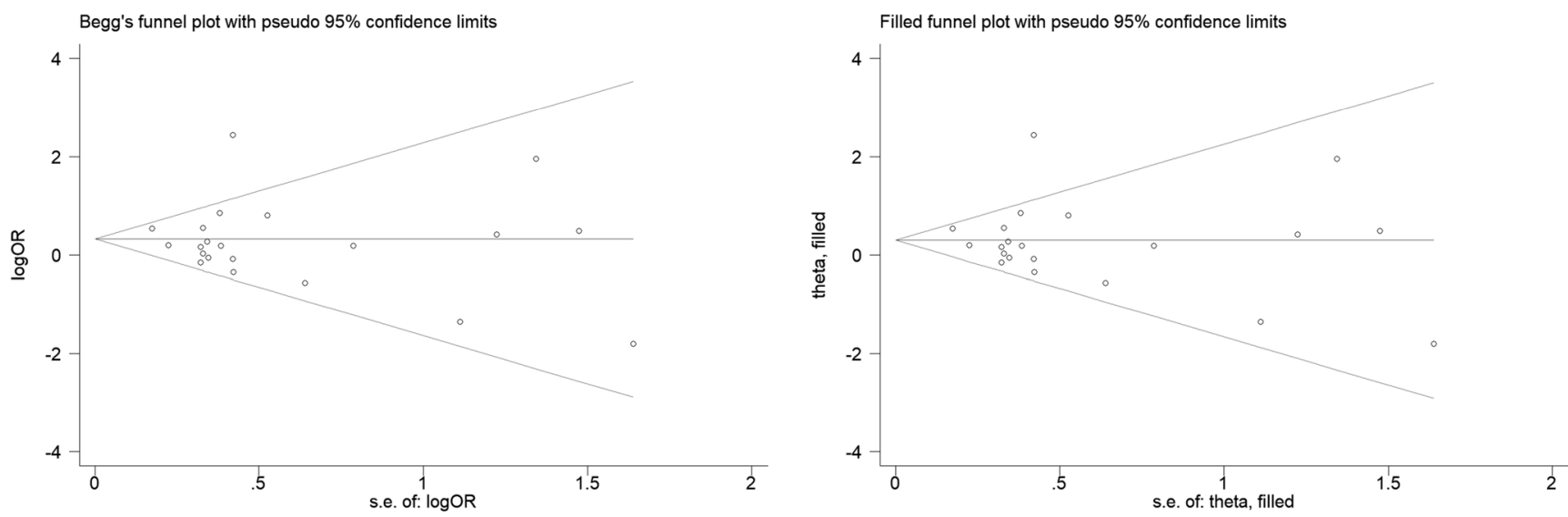

b AGT T704C polymorphism; Dominant genetic model

Fig. 7 Begg's and filled funnel plot of ACE I/D and AGT T704C polymorphisms and PE risk

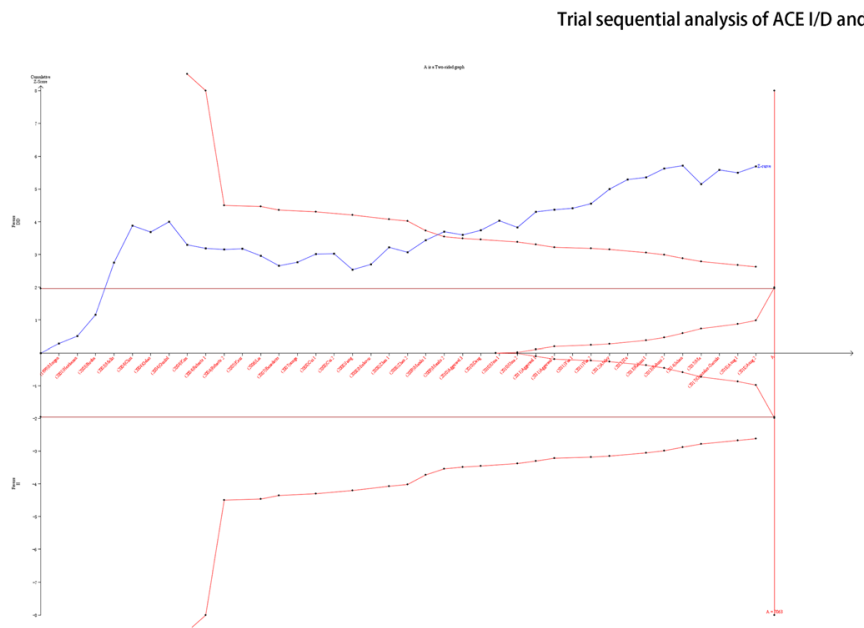

a ACE I/D polymorphism; Homozygote genetic model

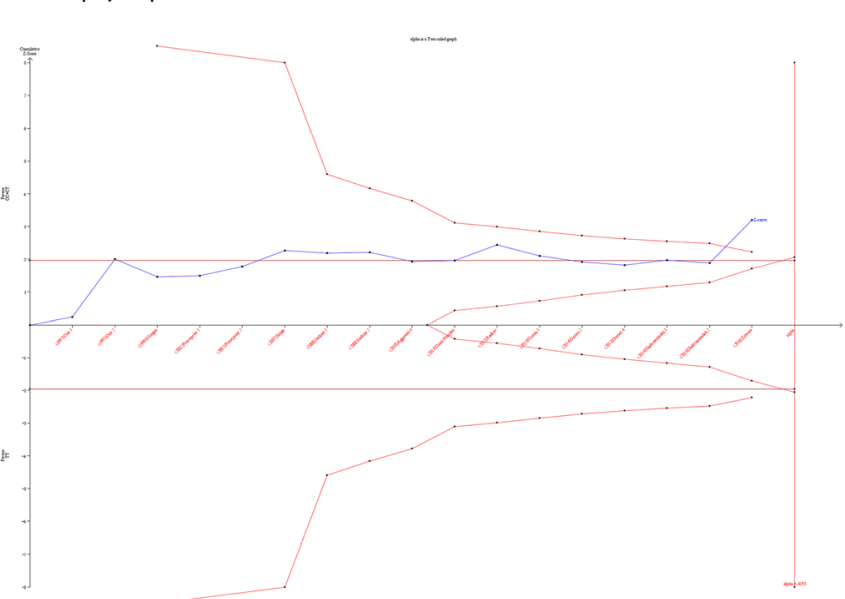

b AGT T704C polymorphism; Dominant genetic model

Fig. 8 Trial sequential analysis of ACE I/D and AGT T704C polymorphisms and PE risk 
ethnicities were published. An increased frequency of AT1R $\mathrm{AC}+\mathrm{CC}$ genotypes in mild preeclamptic women was reported by Rahimi et al [9]. An interaction between the AGT T704C and ACE I/D polymorphisms and the risk of severe preeclampsia or the time onset of PE were observed [7, 8], but these were not analyzed in any former meta-analysis. Drawbacks in terms of high heterogeneity, slack inclusion criteria for subjects from different regions and ethnicities, the lack of evaluation of type 1 error and sample size on significant associations, the vague associations between these polymorphisms and the risk of severe $\mathrm{PE}$, and different onset times of PE greatly aroused our interest. Therefore, we performed an updated meta-analysis with trial sequential analysis to consider the undiscussed above-mentioned issue. Regarding the AT1R A1166C polymorphism, significant associations in mixed race, early-onset, late-onset, and more than 200 patient sample size were discovered; however, only one study was analyzed in these subgroups, implying low representativeness of the AT1R A1166C polymorphism and further studies are essential.

In the overall analysis of the AGT T704C polymorphism, a $33 \%$ increased PE risk of CC + CT genotypes was observed. The 1.26-fold and 1.44-fold increased risk of PE in CT genotypes and CC genotypes, respectively, were also detected compared to TT genotypes. No heterogeneity in the genetic models and the positive results from the trial sequential analysis ensured the stability and reliability of our result. In the subgroup analysis stratified for geography, no significant association was detected; however, increased risks were observed in Caucasoid (the 1.30-fold and 1.28-fold increased risk of CC + CT genotype and CT genotype compared to TT genotype) and Mongoloid (the $60 \%$ increased of $\mathrm{C}$ allele in allelic genetic model; the 4.43-fold increased risk of DD genotype in recessive genetic model). In the severe PE degree subgroup analysis, no association was observed both in either severe or mild PE populations, possibly due to the small sample size, more studies are required. In the more than 200 patient sample size, increased risks were observed in the dominant, recessive, and heterozygote genetic models; however, the relatively small number of included studies in the subgroup indicated that these associations need to be interpreted with caution.

For the ACE I/D polymorphism, the D allele increased the risk of PE compared to I allele by 1.29 -fold; moreover, the DD + DI, DD and DD genotypes increased risk by $17 \%, 52 \%$, and $55 \%$ compared to II, DI + II, and II genotypes, respectively. Significant heterogeneity was observed in the overall analysis. We performed a Galbraith plot analysis to study potential heterogeneity analysis, and after excluding these studies [1, $18,26,32,34,45,46]$, high heterogeneity was significant reduced. We did a comprehensive literature reviewed in these excluded studies; the mixed ethnicities, differences in geography, and patient sample size may be the reasons for the high heterogeneity. Therefore, a full subgroup analysis was conducted. In Asian populations including subjects from China, South Korea, Turkey, Iran, India, and Japan, the increased risk of PE in D allele (allelic genetic model), DD genotype (recessive genetic model), and DD genotype was 1.31-fold, 1.80-fold, and 1.53-fold, respectively. Regarding subjects from Europe (UK, Italy, Greece, and Norway), a $33 \%$ increased risk of PE in D allele (allelic genetic model) and a $68 \%$ increased risk of PE in DD genotypes (homozygote genetic model) were detected, appearing as though the Europeans had more risk of PE than did to Asians. In the subgroup analysis by ethnicity, increased risk of PE was only discovered in Caucasoid population, consistent with results of previous studies [57, 59, 61, 62]. We introduced PE degree and gestational week as subgroups to assess the potential relationships between the ACE I/D polymorphism and severe PE degree and onset time of PE. In the severe PE population, widely increased risks were observed, and we also detected a greater risk of PE than in the mild PE population. However, no significant association was detected for early-onset or lateonset of PE. For the patient sample subgroup analysis, increased risks were also observed.

There were several limitations in this meta-analysis. Firstly, language bias existed in our results; although no language limitation was set, only English and Chinese articles were included. Secondly, the sample size of included studies in the subgroup analysis of PE degree and onset time of PE were relatively small in some groups, implying that our results should be explained with caution. Finally, the potential influence of environment factors on genotype-PE associations is worthy of consideration.

Our results indicated that the AGT T704C and ACE I/D polymorphisms were associated with an increased risk of PE. Increased risks were also observed for the two polymorphisms in subgroups including Asians, Europeans, Caucasoid, and Mongoloid. Furthermore, an increased PE risk with the ACE I/D polymorphism in the severe PE population was also detected. Regarding the AT1R A1166C polymorphism, weak associations were observed and further studies are required.

Funding This work is supported by grants from the National Natural Science Foundation of China (Nos. 81460238) and Jiangxi Province Science and Technology Support Project (Nos. 20142BBG70101, 20171BAB205013). The funder, Shuhui Huang, was responsible for the article.

Data availability The datasets generated during and/or analyzed during the current study are available from the corresponding author on reasonable request.

\section{Compliance with ethical standards}

Competing interests The authors declare that they have no competing interests. 
Ethical approval and informed consent Ethical approval and informed consent were not necessary according to local legislation because of the type of study (meta-analysis).

Open Access This article is licensed under a Creative Commons Attribution 4.0 International License, which permits use, sharing, adaptation, distribution and reproduction in any medium or format, as long as you give appropriate credit to the original author(s) and the source, provide a link to the Creative Commons licence, and indicate if changes were made. The images or other third party material in this article are included in the article's Creative Commons licence, unless indicated otherwise in a credit line to the material. If material is not included in the article's Creative Commons licence and your intended use is not permitted by statutory regulation or exceeds the permitted use, you will need to obtain permission directly from the copyright holder. To view a copy of this licence, visit http://creativecommons.org/licenses/by/4.0/.

\section{References}

1. Gonzalez-Garrido JA, Garcia-Sanchez JR, Tovar-Rodriguez JM, Olivares-Corichi IM. Preeclampsia is associated with ACE I/D polymorphism, obesity and oxidative damage in Mexican women. Pregnancy Hypertens. 2017;10:22-7. https://doi.org/10.1016/j. preghy.2017.04.001.

2. Jeyabalan A. Epidemiology of preeclampsia: impact of obesity. Nutr Rev. 2013;71(suppl 1):S18-25.

3. Yong HE, Murthi P, Brennecke SP, Moses EK. Genetic approaches in preeclampsia. Preeclampsia. Springer; 2018. p. 53-72.

4. Aung M, Konoshita T, Moodley J, Gathiram P. Association of gene polymorphisms of four components of renin-angiotensinaldosterone system and preeclampsia in South African black women. Eur J Obstet Gynecol Reprod Biol. 2017;215:180-7. https://doi. org/10.1016/j.ejogrb.2017.05.011.

5. Zitouni H, Ben Ali Gannoum M, Raguema N, Maleh W, Zouari I, Faleh RE, et al. Contribution of angiotensinogen M235T and $\mathrm{T} 174 \mathrm{M}$ gene variants and haplotypes to preeclampsia and its severity in (North African) Tunisians. J Renin-Angiotensin-Aldosterone Syst. 2018;19(1):1470320317753924. https://doi.org/10.1177/ 1470320317753924.

6. Hall J. Control of sodium excretion by angiotensin II: intrarenal mechanisms and blood pressure regulation. Am J Phys Regul Integr Comp Phys. 1986;250(6):R960-R72.

7. Aung M, Konoshita T, Moodley J, Gathiram P. Association of gene polymorphisms of aldosterone synthase and angiotensin converting enzyme in pre-eclamptic South African Black women. Pregnancy Hypertens. 2018;11:38-43. https://doi.org/10.1016/j.preghy.2017. 12.004 .

8. Shahvaisizadeh F, Movafagh A, Omrani MD, Vaisi-Raygani A, Rahimi Z, Rahimi Z. Synergistic effects of angiotensinogen -217 $\mathrm{G} \rightarrow \mathrm{A}$ and $\mathrm{T} 704 \mathrm{C}$ (M235T) variants on the risk of severe preeclampsia. J Renin-Angiotensin-Aldosterone Syst. 2014;15(2): 156-61. https://doi.org/10.1177/1470320312467555.

9. Rahimi Z, Rahimi Z, Mozafari H, Parsian A. Preeclampsia and angiotensin converting enzyme (ACE) I/D and angiotensin II type-1 receptor (AT1R) A1166C polymorphisms: association with ACE I/D polymorphism. J Renin-Angiotensin-Aldosterone Syst. 2013;14(2):174-80. https://doi.org/10.1177/1470320312448950.

10. Moher D, Liberati A, Tetzlaff J, Altman DG. Preferred reporting items for systematic reviews and meta-analyses: the PRISMA statement. Ann Intern Med. 2009;151(4):264-9 w64.

11. Herraiz I, Llurba E, Verlohren S, Galindo A. Update on the diagnosis and prognosis of preeclampsia with the aid of the sFlt-1/ PlGF ratio in singleton pregnancies. Fetal Diagn Ther. 2018;43(2):81-9. https://doi.org/10.1159/000477903.

12. Goedegebure EAR, Koning SH, Hoogenberg K, Korteweg FJ, Lutgers HL, Diekman MJM, et al. Pregnancy outcomes in women with gestational diabetes mellitus diagnosed according to the WHO-2013 and WHO-1999 diagnostic criteria: a multicentre retrospective cohort study. BMC Pregnancy Childbirth. 2018;18(1): 152. https://doi.org/10.1186/s12884-018-1810-5.

13. ACOG practice bulletin. Diagnosis and management of preeclampsia and eclampsia. Number 33, January 2002. Obstet Gynecol. 2002;99(1):159-67.

14. Stang A. Critical evaluation of the Newcastle-Ottawa scale for the assessment of the quality of nonrandomized studies in meta-analyses. Eur J Epidemiol. 2010;25(9):603-5. https://doi.org/10.1007/ s10654-010-9491-z.

15. Niemeyer H, Musch J, Pietrowsky R. Publication bias in metaanalyses of the efficacy of psychotherapeutic interventions for depression. J Consult Clin Psychol. 2013;81(1):58-74.

16. Duval S, Tweedie R. Trim and fill: A simple funnel-plot-based method of testing and adjusting for publication bias in meta-analysis. Biometrics. 2000;56(2):455-63.

17. Wang X, Cheng W, Ma Y, Zhu J. Vitamin D receptor gene FokI but not TaqI, ApaI, BsmI polymorphism is associated with Hashimoto's thyroiditis: a meta-analysis. Sci Rep. 2017;7:41540. https://doi.org/ 10.1038/srep41540.

18. Ma L, Fan P, Liu XH, He GL, Liu R, Ren RM, et al. Interaction between GNB3 C825T and ACE I/D polymorphisms in preeclampsia. Sichuan Da Xue Xue Bao Yi Xue Ban. 2015;46(1): $118-22$.

19. Jahan P, Deepthi G, Komaravalli PL, Usha RV. A study on the role of HLA-G $14 \mathrm{bp}$ and ACE IN/DEL polymorphisms in preeclamptic South Indian women. Pregnancy Hypertens. 2014;4(2): 164-9. https://doi.org/10.1016/j.preghy.2014.03.002.

20. Groten T, Schleussner E, Lehmann T, Reister F, Holzer B, Danso $\mathrm{KA}$, et al. eNOSI4 and EPHX1 polymorphisms affect maternal susceptibility to preeclampsia: analysis of five polymorphisms predisposing to cardiovascular disease in 279 Caucasian and 241 African women. Arch Gynecol Obstet. 2014;289(3):581-93. https://doi.org/10.1007/s00404-013-2991-9.

21. Song C, Xie S, Wang J, Lian J, Diao B, Tang Y. Association of angiotensinogen gene polymorphisms and angiogenic factors with preeclampsia in Chinese women. Gynecol Obstet Investig. 2013;76(1):64-8. https://doi.org/10.1159/000352070.

22. Radkov OV, Kalinkin MN, Zavarin VV. Genophenotypic analysis of angiotensinogen gene M235T polymorphism and preeclampsia. Bull Exp Biol Med. 2013;154(3):354-6.

23. Kvehaugen AS, Melien O, Holmen OL, Laivuori H, Oian P, Andersgaard AB, et al. Single nucleotide polymorphisms in G protein signaling pathway genes in preeclampsia. Hypertension. 2013;61(3):655-61. https://doi.org/10.1161/hypertensionaha.111. 00331.

24. Coral-Vazquez RM, Romero Arauz JF, Canizales-Quinteros S, Coronel A, Valencia Villalvazo EY, Hernandez Rivera J, et al. Analysis of polymorphisms and haplotypes in genes associated with vascular tone, hypertension and oxidative stress in MexicanMestizo women with severe preeclampsia. Clin Biochem. 2013;46(7-8):627-32. https://doi.org/10.1016/j.clinbiochem.2012. 12.016.

25. Xu YY, Cai QH. Study of Angiotensin-converting enzyme gene polymorphism and angiotensin with Preeclampsia. Jiangxi Med J. 2012;47(10):849-51.

26. Atalay MA, Ozerkan K, Karkucak M, Yakut T, Atik Y, Develioglu $\mathrm{OH}$. Polymorphisms in angiotensin-converting enzyme and glutathione s-transferase genes in Turkish population and risk for preeclampsia. Clin Exp Obstet Gynecol. 2012;39(4):466-9. 
27. Yue BM, He GL, Liu XH. Polymorphisms of angiotensinconverting enzyme gene in pre-eclampsia. Med J West China. 2011;23(05):850-3.

28. Uma R, Forsyth SJ, Struthers AD, Fraser CG, Godfrey V, Murphy DJ. Polymorphisms of the angiotensin converting enzyme gene in early-onset and late-onset pre-eclampsia. J Matern Fetal Neonatal Med. 2010;23(8):874-9. https://doi.org/10.3109/ 14767050903456667 .

29. Aggarwal PK, Jain V, Jha V. Endothelial nitric oxide synthase, angiotensin-converting enzyme and angiotensinogen gene polymorphisms in hypertensive disorders of pregnancy. Hypertens Res. 2010;33(5):473-7. https://doi.org/10.1038/hr.2010.23.

30. Mando C, Antonazzo P, Tabano S, Zanutto S, Pileri P, Somigliana $\mathrm{E}$, et al. Angiotensin-converting enzyme and adducin-1 polymorphisms in women with preeclampsia and gestational hypertension. Reprod Sci. 2009;16(9):819-26. https://doi.org/10.1177/ 1933719109336612.

31. Akbar SA, Khawaja NP, Brown PR, Tayyeb R, Bamfo J, Nicolaides KH. Angiotensin II type 1 and 2 receptors gene polymorphisms in pre-eclampsia and normal pregnancy in three different populations. Acta Obstet Gynecol Scand. 2009;88(5):606-11. https://doi.org/10.1080/00016340902859307.

32. Zhan WX, Zheng JS. Relationship between angiotensin conver ting enzyme gene polymorphism and sever e pr eeclampsia and preeclampsia complicating renal dysfunction. Jiangxi Med J. 2008;43(08):782-4.

33. Miskovic B, Sertic J, Stavljenic-Rukavina A, Stipoljev F. Association of angiotensin-converting enzyme insertion-deletion polymorphism with preeclampsia. Coll Antropol. 2008;32(2): 339-43.

34. Jiang MQ, Fu F. Relationship between angiotensin system related gene polymorphism and pregnancy hypertension disorders. Pract Clin Med. 2008;09(05):25-8.

35. Jenkins LD, Powers RW, Cooper M, Gallaher MJ, Markovic N, Ferrell R, et al. Preeclampsia risk and angiotensinogen polymorphisms M235T and AGT -217 in African American and Caucasian women. Reprod Sci. 2008;15(7):696-701. https://doi.org/10.1177/ 1933719108316984

36. Cui HY, Chen X. The relationship between I/D polymorphism of angiotensin converting enzyme gene and early severe pre-eclampsia. Matern Child Health Care China. 2008;23(11):1545-6.

37. Huang Y, Li YX, Shao JC, Sun LJ. Study on the relationship between gene polymorphism of renin angiotensin system gene and their interaction in preeclampsia. Med Pharm Yunnan. 2007;28(01):8-11.

38. Benedetto C, Marozio L, Ciccone G, Chieppa G, Quaglia M, Matullo G, et al. Synergistic effect of renin-angiotensin system and nitric oxide synthase genes polymorphisms in pre-eclampsia. Acta Obstet Gynecol Scand. 2007;86(6):678-82. https://doi.org/10. 1080/00016340701415269.

39. Li H, Ma YY, Wang LY. The relationship between the ACE gene $\mathrm{I} / \mathrm{D}$ polymorphism and hypertension and renal impairing in preeclamptic patient. Chin J Clin Obsterics Gynecol. 2006;7(6):4113,76 .

40. Seremak-Mrozikiewicz A, Dubiel M, Drews K, Breborowicz GH, Mrozikiewicz PM. 1166C mutation of angiotensin II type 1 receptor gene is correlated with umbilical blood flow velocimetry in women with preeclampsia. J Matern Fetal Neonatal Med. 2005;17(2):117-21. https://doi.org/10.1080/14767050500043400.

41. Kaur R, Jain V, Khuller M, Gupta I, Sherawat BS. Association of angiotensin-converting enzyme gene polymorphism with pregnancy-induced hypertension. Acta Obstet Gynecol Scand. 2005;84(10):929-33. https://doi.org/10.1111/j.0001-6349.2005. 00724.x.

42. Kim YJ, Park MH, Park HS, Lee KS, Ha EH, Pang MG. Associations of polymorphisms of the angiotensinogen M235 polymorphism and angiotensin-converting-enzyme intron 16 insertion/deletion polymorphism with preeclampsia in Korean women. Eur J Obstet Gynecol Reprod Biol. 2004;116(1):48-53. https://doi.org/10.1016/j.ejogrb.2004.01.035.

43. Gurdol F, Isbilen E, Yilmaz H, Isbir T, Dirican A. The association between preeclampsia and angiotensin-converting enzyme insertion/deletion polymorphism. Clin Chim Acta. 2004;341(1-2): 127-31. https://doi.org/10.1016/j.cccn.2003.11.010.

44. Galao AO, de Souza LH, da Costa BE, Scheibe RM, Poli de Figueiredo CE. Angiotensin-converting enzyme gene polymorphism in preeclampsia and normal pregnancy. Am J Obstet Gynecol. 2004;191(3):821-4. https://doi.org/10.1016/j.ajog.2004. 01.047 .

45. Choi H, Kang JY, Yoon HS, Han SS, Whang CS, Moon IG, et al. Association of Angiotensin-converting enzyme and angiotensinogen gene polymorphisms with preeclampsia. J Korean Med Sci. 2004;19(2):253-7. https://doi.org/10.3346/jkms. 2004.19.2.253.

46. Mello G, Parretti E, Gensini F, Sticchi E, Mecacci F, Scarselli G, et al. Maternal-fetal flow, negative events, and preeclampsia: role of ACE I/D polymorphism. Hypertension. 2003;41(4):932-7. https:// doi.org/10.1161/01.hyp.0000063146.40351.ad.

47. Bouba I, Makrydimas G, Kalaitzidis R, Lolis DE, Siamopoulos KC, Georgiou I. Interaction between the polymorphisms of the reninangiotensin system in preeclampsia. Eur J Obstet Gynecol Reprod Biol. 2003;110(1):8-11.

48. Procopciuc L, Jebeleanu G, Surcel I, Puscas M. Angiotensinogen gene M235T variant and pre-eclampsia in Romanian pregnant women. J Cell Mol Med. 2002;6(3):383-8.

49. Heiskanen JT, Pirskanen MM, Hiltunen MJ, Mannermaa AJ, Punnonen KR, Heinonen ST. Insertion-deletion polymorphism in the gene for angiotensin-converting enzyme is associated with obstetric cholestasis but not with preeclampsia. Am J Obstet Gynecol. 2001;185(3):600-3. https://doi.org/10.1067/mob.2001.116722.

50. Morgan L, Foster F, Hayman R, Crawshaw S, Baker PN, Broughton Pipkin F, et al. Angiotensin-converting enzyme insertion-deletion polymorphism in normotensive and preeclamptic pregnancies. J Hypertens. 1999;17(6):765-8.

51. Morgan L, Crawshaw S, Baker PN, Broughton Pipkin F, Kalsheker N. Maternal and fetal angiotensinogen gene allele sharing in preeclampsia. Br J Obstet Gynaecol. 1999;106(3):244-51.

52. Guo G, Wilton AN, Fu Y, Qiu H, Brennecke SP, Cooper DW. Angiotensinogen gene variation in a population case-control study of preeclampsia/eclampsia in Australians and Chinese. Electrophoresis. 1997;18(9):1646-9. https://doi.org/10.1002/elps. 1150180929.

53. Willi C, Bodenmann P, Ghali WA, Faris PD, Cornuz J. Active smoking and the risk of type 2 diabetes: a systematic review and meta-analysis. Jama. 2007;298(22):2654-64. https://doi.org/10. 1001/jama.298.22.2654.

54. Bereketoglu C, Kasap M, Pazarbasi A. Studies on angiotensinconverting enzyme insertion/deletion polymorphism and genotype distributions in Turkish preeclampsia patients. J Pregnancy. 2012;2012:108206-4. https://doi.org/10.1155/2012/108206.

55. Wijaya E, Frith MC, Horton P, Asai K. Finding protein-coding genes through human polymorphisms. PLoS One. 2013;8(1): e54210. https://doi.org/10.1371/journal.pone.0054210.

56. Akdeli N, Riemann K, Westphal J, Hess J, Siffert W, Bachmann HS. A 3'UTR polymorphism modulates mRNA stability of the oncogene and drug target Polo-like Kinase 1. Mol Cancer. 2014;13:87. https://doi.org/10.1186/1476-4598-13-87.

57. Gong FF, Hu CY, Lu SS, Qian ZZ, Feng F, Wu YL, et al. Associations of angiotensin-converting enzyme insertion/deletion, angiotensin II receptor A1166C, and endothelial nitric oxide synthase $4 \mathrm{~b} / \mathrm{a}$ gene polymorphisms with pregnancy hypertensive 
disorders: a meta-analysis. J Clin Hypertens (Greenwich). 2015;17(12):954-62. https://doi.org/10.1111/jch.12606.

58. Zhu M, Zhang J, Nie S, Yan W. Associations of ACE I/D, AGT M235T gene polymorphisms with pregnancy induced hypertension in Chinese population: a meta-analysis. J Assist Reprod Genet. 2012;29(9):921-32. https://doi.org/10.1007/s10815-012-9800-4.

59. Zhong WG, Wang Y, Zhu H, Zhao X. Meta analysis of angiotensin-converting enzyme I/D polymorphism as a risk factor for preeclampsia in Chinese women. Genet Mol Res. 2012;11(3): 2268-76. https://doi.org/10.4238/2012.May.21.1.

60. Zhao L, Dewan AT, Bracken MB. Association of maternal AGTR1 polymorphisms and preeclampsia: a systematic review and metaanalysis. J Matern Fetal Neonatal Med. 2012;25(12):2676-80. https://doi.org/10.3109/14767058.2012.708370.
61. Chen Z, Xu F, Wei Y, Liu F, Qi H. Angiotensin converting enzyme insertion/deletion polymorphism and risk of pregnancy hypertensive disorders: a meta-analysis. J Renin-Angiotensin-Aldosterone Syst. 2012;13(1):184-95. https://doi.org/10.1177/ 1470320311427755 .

62. Serrano NC, Diaz LA, Paez MC, Mesa CM, Cifuentes R, Monterrosa A, et al. Angiotensin-converting enzyme I/D polymorphism and preeclampsia risk: evidence of small-study bias. PLoS Med. 2006;3(12):e520. https://doi.org/10.1371/journal.pmed. 0030520 .

Publisher's note Springer Nature remains neutral with regard to jurisdictional claims in published maps and institutional affiliations. 